\title{
La mise en œuvre de schémas d'usage au service de la construction de l'énoncé en allemand L2
}

\author{
Catherine Felce ${ }^{1}$ \\ ${ }^{1}$ Linguistique, Langues, Parole (LiLPa), EA1339, Université de Strasbourg, France
}

\begin{abstract}
Résumé. L'appropriation de la structure de l'énoncé assertif par des apprenants de l'allemand L2 est généralement considérée du point de vue de la réalisation correcte du positionnement verbal second. Une telle perspective fait de la règle syntaxique $\mathrm{V} 2$ une contrainte que ces derniers doivent non seulement connaitre, mais également appliquer dans leur production langagière. Or, si les connaissances déclaratives permettent de décrire le système linguistique, elles ne sont pas nécessairement converties en une capacité à communiquer qui corresponde aux usages de la langue. Par ailleurs, le placement verbal correct ne suffit à garantir ni la recevabilité d'un énoncé sur le plan communicatif, ni sa conformité par rapport aux principes textuels ou aux préférences discursives de la L2. Dans la présente contribution nous proposons des pistes pour aborder différemment la construction de l'énoncé en allemand L2 : plutôt que de se focaliser sur le placement verbal - certes garant de la correction syntaxique des phrases - il s'agit de familiariser les apprenants avec des usages de l'ouverture qui soient en adéquation avec le contexte communicatif et textuel dans lequel s'insèrent les énoncés produits.
\end{abstract}

\begin{abstract}
For learners of GFL (or German as L2), learning how to produce an assertive is often considered from the perspective of the correct verb placement in the second position. The V2 rule constitutes a constraint that has to be learned and applied in the language production. Thus, if declarative knowledge is related to the description of the linguistic system, it fails to be automatically converted into a communicative ability corresponding to language use. Furthermore, correct verbal placement alone cannot make a sentence communicatively acceptable or conform according to the textual principles or the discursive preferences of the L2. In the article, we propose a different way to teach (and learn) how to structure a declarative sentence in German L2: Instead restrictively focusing on the correct verbal placement -which can only be relevant for syntactic correctness - we're seeking to make learners familiar with ways to start a sentence in order to fit to the communicative and textual context in which the produced utterances take place.
\end{abstract}




\section{Introduction}

Pour les apprenants de L2, la mise en ordre des constituants au sein de la chaine linéaire que constitue un énoncé déclaratif en allemand - langue typologiquement distincte du français - recèle bien des difficultés. Non seulement le positionnement verbal est garant de la correction syntaxique des phrases, mais la réception adéquate des messages produits dépend quant à elle de l'interprétation qui sera faite de la hiérarchisation des constituants. Enfin, pour que la production langagière soit considérée comme conforme à la langue cible, celle-ci doit correspondre aux principes pragmatiques et textuels qui se réalisent à travers différentes options de linéarisation. Dans ce faisceau de paramètres, l'enjeu du positionnement verbal correct semble pourtant à lui seul focaliser toute l'attention, que ce soit dans les pratiques enseignantes, ou dans les travaux acquisitionnistes initiés par les recherches de Pienemann.

La question de l'ordre des mots en allemand est en effet bien connue des chercheurs en acquisition des langues; elle a fait l'objet, dès le début des années 80 , de publications de référence menées dans le cadre du projet ZISA ${ }^{1}$ (Meisel, Clahsen \& Pienemann, 1981; Pienemann, 1984). La mise en évidence d'une séquence développementale, découpée en une succession de stades hiérarchisés, et caractéristique de la progression des apprenants dans leur appropriation de l'ordre des mots a marqué la discipline dans ses débuts. Si ces travaux se sont dans un premier temps essentiellement consacrés à l'acquisition langagière d'adultes migrants en milieu naturel, la mise en lumière d'un tel itinéraire de développement a contribué à interroger, dans des contextes d'apprentissage guidé, la pertinence de l'enseignement grammatical, considéré comme moyen de forcer l'acquisition de certaines formes. Pienemann (1989) a ainsi avancé l'idée que seules les formes que l'apprenant est cognitivement prêt à acquérir, en fonction d'un stade de développement atteint, sont enseignables (Teachability Hypothesis) - hypothèse selon laquelle il s'avère illusoire de chercher à forcer l'acquisition de structures trop éloignées du stade auquel se situent les apprenants. Différentes études, menées par la suite dans des contextes institutionnels (Ballestracci, 2010; Boss \& Jansen, 2003; Diehl, Christen, Leuenberger, Pelvat, \& Studer, 2000; Ellis, 1989) et mettant en jeu différentes langues, ont confirmé la robustesse de la séquence développementale relative à l'ordre des mots, sans toutefois chercher à remettre en cause l'enseignement formel centré sur le positionnement verbal.

Dans le cadre d'une recherche menée avec des apprenants francophones scolarisés dans le secondaire, nous avons développé des propositions didactiques permettant d'aborder différemment la construction de l'énoncé assertif en début d'apprentissage. Il ne s'agit pas d'ignorer la règle du positionnement verbal mais de la subordonner à des réalisations conformes de l'attaque de l'énoncé qui font entrer la fonction de la position préverbale dans le champ d'investigation. Ces propositions ont une visée praxéologique et sont destinées à familiariser les apprenants à l'usage de constructions prototypiques qui impliquent une réalisation de l'Inversion ${ }^{2}$. Plutôt que de réduire ce phénomène à une règle positionnelle prescriptive, l'usage de constructions de type X-Verbe-Sujet, motivé par le cadre discursif fourni par des tâches, nous semble susceptible de favoriser la mise en place d'habitudes ou de préférences pour l'ouverture distinctes du français.

\footnotetext{
${ }^{1}$ Zweitspracherwerb Italienischer und Spanischer Arbeiter. [L'acquisition de langue seconde par des travailleurs italiens et espagnols.]

${ }^{2}$ Il s'agit ici du phénomène particulier décrit dans la hiérarchie des stades d'acquisition de l'ordre des mots et qui consiste à antéposer une expression adverbiale à l'ouverture de l'énoncé, entrainant un positionnement postverbal du sujet. L'emploi de ce terme étant problématique pour les germanistes, nous faisons ici le choix de l'écrire avec une majuscule pour souligner que nous employons ce terme exclusivement en référence au phénomène évoqué plus haut et en aucun cas pour décrire la structure de la phrase allemande.
} 
Nous nous sommes par conséquent éloignée des analyses syntaxiques - qui prévalent à la fois sur le plan développemental ${ }^{3}$ ou grammatical (position catégorielle des éléments dans la phrase) - pour prendre en compte des approches théoriques, qui pour diverses qu'elles soient, se rejoignent dans l'intérêt qu'elles manifestent pour la langue dans différentes situations d'emploi. Des notions issues de la linguistique textuelle ont ainsi été mises à profit pour comprendre le rôle de l'ouverture dans la succession des énoncés et la production langagière a été vue comme un agencement d'unités informationnelles soumis aux préférences de la langue cible et aux enjeux communicatifs sous-jacents.

Notre travail vise à proposer des pistes pour la mise en œuvre de ces nouvelles connaissances dans l'intervention enseignante et pour le guidage de la production langagière des apprenants: comment faire entrer dans les pratiques enseignantes des savoirs mieux à même de décrire le fonctionnement des usages que ne le font des règles prescriptives liées au système ? Comment opérationnaliser les savoirs théoriques dans des activités qui permettent aux apprenants de s'approprier l'usage stratégique du champ initial (Vorfeld) ? Une production contrôlée, destinée à les guider vers des réalisations de l'ouverture conformes à l'usage, est-elle enfin susceptible de s'accompagner d'acquisitions syntaxiques spécifiques - en l'occurrence d'une réalisation correcte de l'Inversion?

\section{De nouvelles connaissances au service de la description des usages et de leur mise en œuvre dans la classe de langue}

\subsection{Le positionnement verbal second dans l'énoncé : de la difficulté d'acquérir une règle enseignée}

A la fois langue à directionnalité vers la gauche remontant de la position finale de la base verbale (Fabricius-Hansen, 2010) et langue dite à verbe second, l'allemand se caractérise par la position fixe des éléments verbaux qui constituent une sorte de parenthèse ou de «pince verbale »: les éléments non fléchis apparaissent en position finale et la forme fléchie du verbe occupe la deuxième position. Le verbe second fait office de borne et délimite un espace à sa gauche (le Vorfeld ou champ initial) en réduisant l'occupation de ce dernier à un seul constituant (une seule unité informationnelle), de nature ou de fonction variée. Une sélection doit donc s'opérer pour le choix de l'élément positionné dans le Vorfeld.

Tableau 1. Modèle topologique de la phrase allemande avec la « pince verbale ».

\begin{tabular}{|c|c|c|c|}
\hline \multicolumn{4}{|c|}{$\begin{array}{l}\text { Ich will mit Peter nach Berlin fahren } \\
\text { [Je veux avec Peter à Berlin aller] }\end{array}$} \\
\hline & [parent & ce verbale] & ammer \\
\hline $\begin{array}{l}\text { Vorfeld - [champ initial] } \\
\text { Attaque de l'énoncé }\end{array}$ & & Mittelfeld - [champ médian] & \\
\hline $\mathbf{X}$ & $\mathbf{V} 2$ & & V END \\
\hline $\begin{array}{l}\text { Ich } \\
{[\mathrm{Je}}\end{array}$ & $\begin{array}{c}\text { will } \\
\text { veux }\end{array}$ & $\begin{array}{cc}\text { mit Peter } & \text { nach Berlin } \\
\text { avec Peter } & \text { à Berlin }\end{array}$ & $\begin{array}{c}\text { fahren } \\
\text { aller] }\end{array}$ \\
\hline
\end{tabular}

\footnotetext{
${ }^{3}$ On peut se reporter à l'ouvrage de Pienemann et Keßler (2011) pour une synthèse de la théorie de la Processabilité (Processabilty Theory) et pour une description du développement graduel des procédures syntaxiques responsables de l'échange d'informations grammaticales, d'abord au sein d'un syntagme, puis entre les constituants de l'énoncé.
} 


\begin{tabular}{|c|c|c|c|}
\hline $\begin{array}{c}\text { Nächste Woche } \\
\text { [la semaine prochaine }\end{array}$ & $\begin{array}{c}\text { will } \\
\text { veux }\end{array}$ & $\begin{array}{c}\text { ich nach Berlin } \\
\text { je à Berlin }\end{array}$ & $\begin{array}{c}\text { fahren } \\
\text { aller] }\end{array}$ \\
\hline $\begin{array}{c}\text { Mit Peter } \\
\text { [Avec Peter }\end{array}$ & $\begin{array}{c}\text { will } \\
\text { veux }\end{array}$ & $\begin{array}{c}\text { ich nach Berlin } \\
\text { à Berlin }\end{array}$ & $\begin{array}{c}\text { fahren } \\
\text { aller ] }\end{array}$ \\
\hline
\end{tabular}

Généralement, les descriptions des manuels scolaires n'accordent pas plus d'attention à cette variété possible à l'ouverture et à la compétition qui existe entre différents constituants. Elles se contentent de présenter le positionnement verbal second dans l'énoncé assertif comme une règle d'or, destinée à être enseignée dès les débuts de l'apprentissage. On peut lire ainsi dans différents manuels destinés à la première année d'apprentissage :

Dans la proposition déclarative, le verbe conjugué occupe la $2^{\mathrm{e}}$ place, c'est-à-dire qu'il est le deuxième groupe à avoir une fonction dans la phrase. La $1^{\text {re }}$ place est occupée par le sujet ou d'autres compléments (Haldenwang, Lozachmeur, Pavan, \& Suissa, 2009, p. 124).

Dans une déclarative, le verbe conjugué occupe toujours la $2^{\mathrm{e}}$ place. Lorsque la première place est occupée par un complément le sujet se place après le verbe (Faure-Paschal, Hoock-Douilly, Rakotondravao, \& Wegener, 2013, p. 30)

Dans la proposition déclarative (tout comme dans la proposition interrogative en W-), le verbe conjugué est toujours à la deuxième place. La première place n'est pas obligatoirement occupée par le sujet ! (Goldmann, Jacqueroud, \& Robert, 2013, p. 32).

Pour simple qu'elle soit dans sa formulation, la règle relative au positionnement verbal second et à la réalisation de l'Inversion l'est moins dans son application par des apprenants de L2 débutant l'apprentissage de la langue et dotés d'un système linguistique dans lequel prévalent d'autres préférences : le français permet une accumulation de constituants avant le verbe et réserve généralement au sujet la position préverbale.

A la suite des travaux de Pienemann et de l'hypothèse qu'il formule quant à «l'enseignabilité » de certaines formes, des études menées dans différents contextes d'apprentissage guidé et à partir de différentes langues sources ont en effet montré les difficultés rencontrées par les apprenants pour acquérir la règle de l'Inversion dans l'ordre prévu par la séquence d'enseignement. A celle-ci se substitue en effet un ordre naturel d'acquisition dans lequel chaque stade conditionne l'accession au stade supérieur.

Tableau 2. Hiérarchie des stades d'acquisition mis en évidence dans le cadre de l'étude ZISA d'après Pienemann (1999, p. 45).

\begin{tabular}{|l|l|}
\hline $\begin{array}{l}\text { (1) (2) Stade } \mathbf{x}=\text { ordre canonique } \\
\text { (Canonical Order) }\end{array}$ & $\begin{array}{l}\text { die kinder spielen mim ball } \\
\text { [the children play with the ball] }\end{array}$ \\
\hline $\begin{array}{l}\text { (3) Stade } \mathbf{x}+\mathbf{1}=\text { positionnement } \\
\text { adverbial devant le verbe } \\
\text { (Adverb Preposing) (ADV) }\end{array}$ & $\begin{array}{l}\text { da kinder spielen } \\
\text { [there children play] }\end{array}$ \\
\hline $\begin{array}{l}\text { (4) Stade } \mathbf{x}+\mathbf{2}=\text { séparation des éléments } \\
\text { fléchis et non fléchis } \\
(\text { Verb Separation) } \text { (SEP) }\end{array}$ & $\begin{array}{l}\text { alle kinder muss die pause machen } \\
\text { [all children must the break have] }\end{array}$ \\
\hline $\begin{array}{l}\text { (5) Stade } \mathbf{x}+\mathbf{3}=\text { Inversion } \\
\text { Inversion) }(\mathrm{INV})\end{array}$ & $\begin{array}{l}\text { dann hat sie wieder die knoch gebringt } \\
\text { [then has she again the bone bringed] }\end{array}$ \\
\hline $\begin{array}{l}\text { (6) Stade } \mathbf{x}+\mathbf{4}=\text { verbe final dans la } \\
\text { subordonnée } \\
(\text { Verb Final) (V-END) }\end{array}$ & $\begin{array}{l}\text { er sagt, dass er nach hause } \text { kommt } \\
\text { [he says that he home comes] }\end{array}$ \\
\hline
\end{tabular}

${ }^{4}$ Nous avons laissé les exemples en anglais tels qu'ils sont donnés par Pienemann. 
Ces nombreuses études ont confirmé la séquence développementale illustrée dans le tableau 2, montrant par là la difficulté à faire coïncider séquence d'apprentissage (d'appropriation) et séquence d'enseignement (Véronique, 2009). Pienemann (1984, 1989) a montré que les apprentissages étaient contraints par des procédures de traitement syntaxiques sous-jacentes et que la hiérarchie des stades ne pouvait être modifiée par l'instruction formelle. Voulant comparer acquisition naturelle et scolaire, Ellis (1989) aboutit au même constat, à savoir que l'acquisition de l'ordre des mots est soumise à des mécanismes internes qui ne se laissent pas infléchir par l'input grammatical fourni. Plus récemment, ces travaux ont été complétés par d'autres recherches (Ballestracci, 2010; Boss \& Jansen, 2003; Cedden \& Aydın, 2007) qui, en dépit de quelques nuances, rejoignent la hiérarchie des stades établie suite à ZISA et les prédictions de Pienemann quant à la disponibilité progressive de procédures nécessaires au traitement de structures syntaxiques particulières. La plus marquante de ces études est celle menée par Diehl et ses collègues au sein des écoles genevoises (projet $D i G S^{5}$ ) (Diehl et al., 2000) et dont les conclusions ont remis en question les progressions d'enseignement institutionnellement établies.

Par ailleurs, d'autres travaux acquisitionnistes se sont penchés sur les productions d'apprenants avancés - voire très avancés - et ont souligné le fait que la correction morphosyntaxique ne constitue pas une condition suffisante pour atteindre un niveau de compétence proche de celui de locuteurs natifs. Les apprenants peuvent bien disposer de connaissances morphosyntaxiques qui garantissent la correction des énoncés qu'ils produisent, celles-ci ne leur permettent cependant pas de développer une compétence communicative jugée conforme par des germanophones. Dans le cas d'apprenants suédophones par exemple, dont la L1 est typologiquement proche de l'allemand, Bohnacker et Rosén (2008) relèvent le manque d'expressions adverbiales à l'ouverture, et notamment de formes pronominales spécifiques ${ }^{6}$ qui jouent un rôle prépondérant dans le maintien de la référence au fil des énoncés successifs en leur conférant cohérence et cohésion. Dalmas (2005) fait un constat similaire face à des productions d'apprenants francophones, qui trahissent un manque de compétence textuelle à travers des choix arbitraires à l'ouverture et une absence de cohésion chronologique ou argumentative entre les énoncés.

Ces observations renvoient à deux niveaux de la production langagière ${ }^{7}$ : au niveau de l'encodage d'une part, les formes langagières utiles au marquage des relations référentielles dans la linéarité des énoncés et du texte s'avèrent absentes des productions observées. En amont d'autre part, au niveau de la conceptualisation même du message, la mise en ordre des unités informationnelles ne correspond pas à un schéma informationnel pertinent en langue cible. Pour von Stutterheim et ses collègues, il manque à de telles productions le «bon accent cognitif» (von Stutterheim \& Carroll, 2005); autrement dit, les écarts observables ne sont pas à rapporter à des problèmes d'encodage mais à une conceptualisation qui demeure influencée par les habitudes et les préférences de la L1.

\footnotetext{
${ }^{5}$ Deutsch in Genfer Schulen. [L'allemand dans les écoles genevoises.]

${ }^{6}$ Il s'agit d'expressions pronominales construites à partir d'un élément anaphorique « $d a$ » et d'une préposition : par exemple danach [après ça=le moment mentionné précédemment] ou encore daneben [à côté de ça=le repère posé en amont].

Telle qu'elle est modélisée dans le modèle de Levelt (1989) qui décompose la production langagière en trois étapes successives : conceptualisation d'un message préverbal, encodage grammatical et phonologique destiné à la mise en mots de ce message conceptuel et articulation qui correspond à la réalisation phonétique du message préalablement encodé.
} 


\subsection{Au-delà des règles positionnelles: quels enjeux stratégiques à l'ouverture?}

Dans un article consacré à la prise en compte de la compétence textuelle dans les apprentissages, Dalmas $(2005$, p. 97) déplore la priorité trop souvent accordée aux règles, c'est-à-dire aux aspects conventionnels de la langue, aux dépens des paramètres stratégiques qui sous-tendent la production langagière. A l'ouverture, il n'y a en allemand de contrainte ni catégorielle, ni syntaxique - seule une accumulation de différents éléments qui ne constituent pas un ensemble informationnel homogène est impossible. Le Vorfeld ne pouvant, en tant qu'espace borné, accueillir qu'un constituant, il est nécessaire d'opérer une sélection entre les candidats potentiels à l'occupation du champ initial et la résolution de cette compétition se fonde sur des critères informationnels et textuels. C'est en ce sens que le Vorfeld représente une position stratégique dans l'énoncé et que le constituant initial remplit des fonctions essentielles, non seulement au sein de la phrase mais aussi du discours / texte. Nous retiendrons trois de ces fonctions pour la présente contribution, illustrées par les exemples suivants de (1) à (4) que nous empruntons à Poitou (2010) :

(1) Ich bin gestern mit Maria ins Theater gegangen. [Je suis allé au théâtre avec Maria.]

(2) Gestern bin ich mit Maria ins Theater gegangen. [Hier, je suis allé au thêâtre avec Maria.]

(3) Dieses Stück von Brecht habe ich schon zehnmal gesehen. [Cette pièce de Brecht, je l'ai déjà vue 10 fois.]

(4) Ins Theater bin ich gestern mit Maria gegangen, nicht ins Kino. [Je suis allé au théâtre hier avec Maria, pas au cinéma.]

L'attaque de l'énoncé, c'est-à-dire l'élément mentionné en premier dans la châne linéaire, participe tout d'abord de la répartition et de la hiérarchisation des contenus informationnels véhiculés. Un élément connu ou donné, car mentionné en amont, ou encore un savoir préalablement activé sert de point de départ à l'énoncé ; il est ensuite complété par de nouvelles unités informationnelles qui sont apportées en fin de proposition. En allemand, la progression informationnelle qualifiée de non marquée est ainsi caractérisée par une progression de type topique - focus qui fait des éléments topicaux des amorces de l'énoncé. En tant qu'élément topical, le sujet apparait relativement fréquemment en position préverbale comme en (1), sans toutefois que cet espace ne lui soit réservé.

Si l'on considère en effet différentes expressions situatives (locatives ou temporelles notamment) comme relevant également du topique ${ }^{8}$, il apparait qu'en allemand, ces expressions concurrencent le sujet dans l'occupation du Vorfeld (VF) comme en (2). L'échelle préférentielle établie par Speyer (2008) confirme la prévalence de cadratifs ${ }^{9}$ à l'ouverture (Scene-setting $V F$ ) lorsque ceux-ci sont dotés d'une valeur informationnelle ou fonctionnelle supérieure à celle des autres candidats topicaux, qu'il s'agisse de topiques contrastifs (Contrast VF) - qui se comprennent dans une relation d'opposition, de restriction ou de rectification par rapport à des éléments mentionnés ou activés au préalable, comme en (4) - ou du topique, sujet de l'énoncé (Topic VF).

\footnotetext{
${ }^{8}$ Lahousse (2003, p. 121) rappelle notamment que Chafe adopte une définition large de la notion de topique qui lui reconnait la propriété de poser un cadre spatio-temporel dans lequel s'insère le contenu propositionnel.

${ }^{9}$ En référence ici à Charolles (2003) pour qui les adverbiaux cadratifs constituent des topiques scéniques.
} 


\section{SCENE-SETTING-VF $>>$ CONTRAST-VF $>>$ TOPIC-VF}

Fig. 1. Hiérarchie des expressions candidates à l'occupation du Vorfeld (VF), selon Speyer (2008, p. 287).

Au sein d'un texte, considéré comme une suite d'énoncés, les expressions temporelles ou spatiales positionnées à l'ouverture peuvent par ailleurs marquer des transitions entre énoncés par le biais d'une succession d'intervalles repérés. Le déploiement informationnel est dès lors guidé par un décalage (temporel ou spatial) qui s'opère d'un intervalle repéré à un autre (von Stutterheim, 1997).

Enfin, les éléments positionnés en amont du verbe assurent le lien avec le pré-texte à travers la reprise d'un contenu propositionnel activé dans l'énoncé qui précède, en (3); une continuité propositionnelle est ainsi explicitement marquée à travers des phénomènes de reprise anaphorique et la cohésion inter-phrastique est d'autant plus grande qu'elle s'accompagne d'une proximité topologique entre les éléments mis de la sorte en relation (Vinckel-Roisin, 2012).

Si ces fonctions associées au Vorfeld sont désormais bien connues des linguistes, elles n'ont cependant pas su pénétrer les connaissances destinées aux apprenants. Moins univoques, et par conséquent en apparence moins simples que la règle du placement verbal second, elles nécessitent de dépasser le cadre de la phrase et n'ont pas la rigidité de la règle prescriptive. Elles se recomposent au contraire selon le contexte et les intentions communicatives du locuteur; dans la mesure néanmoins où elles participent de l'agencement, de la hiérarchisation et de la progression informationnelle, elles sont liées à des usages et à des préférences auxquels il est nécessaire de familiariser les apprenants.

A cette fin, et en accord avec l'arrière-plan théorique mentionné, l'usage apparait comme une notion dont il convient de réévaluer la valeur épistémologique (Legallois \& François, 2011) et à partir de laquelle peuvent se développer d'autres connaissances sur la langue. Celles-ci permettent de prendre la mesure des paramètres cognitifs liés au traitement d'unités informationnelles plus ou moins complexes, et de comprendre les aspects discursifs, conversationnels ou textuels à travers lesquels s'actualisent différents usages de la langue. Ces apports théoriques pluriels nourrissent aussi bien les recherches linguistiques que celles sur l'acquisition des langues secondes (RAL) ou de la didactique des langues. François et Legallois font remarquer à juste titre le caractère fédérateur du terme usage, autour duquel s'agrègent différents domaines théoriques, et qui, nous le pensons, offre également une passerelle vers les pratiques de terrain et la conception d'activités d'apprentissage en accord avec la perspective actionnelle mise en avant par le Cadre Européen Commun de Référence pour les Langues (CECRL). Dans le travail que nous avons mené, aussi bien en tant qu'enseignante qu'en tant que chercheuse, l'usage peut être convoqué dans les deux acceptions que Legallois et François (2011) attribuent au terme, à savoir l'usage-emploi d'une part, qui désigne la pratique, l'utilisation de la langue dans des contextes et des situations variées et que l'on peut également rapporter à la pratique langagière en classe de langue (output practice); et l'usage-habitude d'autre part, qui fait plus largement référence aux usages compris comme préférences ou routines et qui sont majoritairement observables dans les productions des locuteurs d'une langue donnée.

\subsection{Des usages de la langue comme soutien à l'appropriation}

La reconnaissance de cette double acception associée au terme d'usage est d'importance pour l'enseignement des langues qui fait de la pratique un vecteur privilégié de l'apprentissage (Muranoi, 2007), et plus spécifiquement dans le cadre de l'approche par 
tâches (Ellis, 2003; Skehan, 2007) et des orientations du CECRL qui fait de l'apprenant un locuteur / usager de L2 à part entière.

Dans le contexte institutionnel, de surcroit exolingue, le travail sur l'input et la production langagière en langue cible s'inscrivent dans le cadre de la classe de langue. C'est dans cet espace que prennent place les activités de repérage de constructions particulières dans les données langagières fournies, ainsi que les activités de production plus ou moins guidée ou contrôlée, destinées à favoriser la manipulation de schémas spécifiques - et, au-delà, leur fixation mémorielle et leur routinisation. Chaque séquence pédagogique est ainsi conçue comme un ensemble organisé de micro-tâches, déclinées en activités de réception et de production et visant le repérage (noticing), la production contrôlée (controlled output practice), la fixation en mémoire de constructions spécifiques et enfin leur rappel dans une production plus libre permettant le réinvestissement de ces mêmes constructions ou de variantes inédites. La répétition comme la fréquence de certaines associations contribuent à banaliser l'usage des associations produites tout en favorisant leur rappel dans des situations similaires.

Dans une telle organisation des activités, l'usage est d'abord compris comme emploi et manipulation des moyens langagiers déjà acquis et de ceux mis à disposition; ce travail de mise en œuvre doit conduire à la mise en place d'habitudes ou de préférences spécifiques à la L2 chez les apprenants. Préférences qui font que, de l'usage-emploi, émerge un usage plus routinier de constructions distinctes de celles de leur L1.

Nous avons cherché à reformuler des objectifs d'apprentissage pour en faire des questions liées à des enjeux qui nous semblent cruciaux pour des apprenants débutant l'apprentissage de l'allemand. Il s'agit entre autres de :

- la hiérarchisation informationnelle et des variations à l'attaque en fonction des questions sous-jacentes,

- la chronologie temporelle des événements,

- l'établissement de la cohésion entre énoncés successifs.

Il s'agit là d'aspects susceptibles d'intervenir dans différentes tâches pouvant être proposées en début d'apprentissage, comme par exemple l'échange d'informations sur les activités extra-scolaires, ou encore le récit d'une journée d'école. Ces aspects ne sont par ailleurs pas dissociables les uns des autres, la hiérarchie des places dans l'énoncé, comme la succession des éléments chronologiques, participent toutes deux de la cohésion interphrastique. Et cette dernière, à travers l'occupation du champ initial, garantit en retour la cohérence de l'ensemble. On voit là que se posent des questions qui n'entrent pas dans le cadre de la grammaire traditionnelle et qu'il s'agit de faire comprendre aux apprenants, audelà d'une terminologie bien souvent abstraite, par quels moyens des formes langagières permettent la restitution d'un événement à partir des fonctions précises : la focalisation vers l'information nouvelle, la chronologie ou encore le maintien au fil du discours / texte de différents contenus activés.

\section{Constitution du corpus}

\subsection{Participants et type de tâches recueillies}

Les apprenants que nous avions en charge en tant qu'enseignante étaient des élèves scolarisés dans le secondaire, dans un collège de la proche banlieue parisienne sans classement particulier. Ils ont débuté en $6^{\text {ème }}$ leur apprentissage de l'allemand dans un dispositif dit bilangue ${ }^{10}$ qui leur permettait de poursuivre l'apprentissage de l'anglais -

${ }^{10}$ Dans un tel dispositif, les élèves de $6^{\text {ème }}$ apprennent deux langues dès la première année du collège. 
langue que la majorité des élèves avaient suivie durant 3 ans à l'école élémentaire - tout en débutant une autre langue, en l'occurrence l'allemand. Seuls 5 élèves avaient pu bénéficier en CM2 d'une initiation à l'allemand en plus des habituels cours d'anglais. Enfin, un seul élève avait quant à lui suivi exclusivement un enseignement d'allemand du CE2 au CM2.

Tableau 3. Données synthétiques sur les participants.

\begin{tabular}{|c|c|c|}
\hline & $1^{\text {ère }}$ année de recueil - classe de & $2^{\text {ème }}$ année de recueil - classe de \\
\hline $\begin{array}{l}\text { Composition } \\
\text { du groupe } \\
\text { classe }\end{array}$ & $\begin{array}{l}\text { - } 25 \text { élèves } \\
\text { - âgés de } 10 \text { à } 12 \text { ans } \\
-8 \text { garçons / } 17 \text { filles }\end{array}$ & $\begin{array}{l}\text { - } 24 \text { élèves } \\
\text { - âgés de } 11 \text { à } 13 \text { ans } \\
\text { - } 7 \text { garçons / } 17 \text { filles }\end{array}$ \\
\hline
\end{tabular}

Les productions de ces participants ont été recueillies durant les deux premières années d'apprentissage, en $6^{\text {ème }}$ et en $5^{\text {ème }}$, à partir de tâches intégrées dans les séquences d'enseignement et destinées à évaluer les acquis, généralement en fin de séquence. Dans les premières années d'apprentissage, le développement des compétences langagières correspondant aux niveaux A1 et $\mathrm{A} 2$ se fait à travers des activités liées à l'environnement immédiat des apprenants : l'école, la famille, les loisirs, entre autres thèmes. En relation avec ces thématiques, nous avons orienté notre intervention enseignante en classe de telle sorte que les apprenants aient à manipuler des schémas pertinents pour la réalisation de tâches relevant des domaines cités.

Les données ${ }^{11}$ présentées dans cette contribution sont issues de trois tâches : deux d'entre elles ont été proposées en classe de $6^{\text {ème }}$ (wochenplan et kino) et la troisième en cours d'année de $5^{\text {ème }}$ (tagesablauf). Il est important de souligner que ces tâches, décrites dans le tableau 4, n'ont pas spécialement été élaborées à des fins de recherche mais qu'elles ont fait partie des activités normalement menées en classe. Elles ont été effectuées dans le cadre de la classe pendant les horaires de cours et n'ont demandé ni travail supplémentaire, ni présence de la part des apprenants en dehors des horaires habituels. Elles ont enfin été proposées à l'issue de différentes séquences d'enseignement / apprentissage et ont permis de mesurer la manière dont étaient réinvesties des constructions ayant fait l'objet de repérage, d'explications ou d'entraînements au sein d'activités préalablement menées dans les séquences d'enseignement proposées.

Tableau 4. Synthèse des tâches sélectionnées pour la présente contribution.

\begin{tabular}{|l|l|c|}
\hline wochenplan & \multicolumn{1}{|c|}{ Caractéristiques } & $\begin{array}{l}\text { Nombre } \\
\text { d'énoncés }\end{array}$ \\
\hline & $\begin{array}{l}\text { Ces loisirs de la semaine à partir duquel les échanges vont avoir } \\
\text { lieu. } \\
\text { Objectif communicatif } \\
\text { du temps de manière à compléter un planning vierge et reconstituer } \\
\text { l'emploi du temps de ses activités hebdomadaires à partir des } \\
\text { informations recueillies. } \\
\text { - Tâche d'entraînement ciblée sous forme d'interaction orale en } \\
\text { binôme. } \\
\text { - Enregistrement audio effectué par les apprenants pendant leur } \\
\text { interaction. }\end{array}$ & \\
\hline
\end{tabular}

\footnotetext{
${ }^{11}$ Ce corpus de productions d'apprenants a été constitué dans le cadre de notre thèse de doctorat (Felce, 2015).

${ }^{12}$ Il s'agit ici du résultat communicatif auquel la tâche doit aboutir. Ellis (2003) distingue en effet le résultat opérationnel auquel une tâche doit aboutir (outcome) de l'objectif d'apprentissage visé par l'enseignant par le biais de la réalisation de la tâche (aim).
} 


\begin{tabular}{|c|c|c|}
\hline kino & $\begin{array}{l}\text { Chaque partenaire dispose d'un emploi du temps élaboré par } \\
\text { l'enseignante avec différentes activités. } \\
\text { Objectif communicatif: Trouver un créneau commun pour aller } \\
\text { au cinéma et expliquer pourquoi certains créneaux ne conviennent } \\
\text { pas. } \\
\text { - Tâche d'évaluation de la production orale en interaction. } \\
\text { - Enregistrement audio effectué par les apprenants pendant leur } \\
\text { interaction. }\end{array}$ & 154 \\
\hline tagesablauf & $\begin{array}{l}\text { Les apprenants disposent de vignettes pour différents adolescents } \\
\text { dans lesquelles des horaires et des activités sont mis en regard }{ }^{13} \text {. Ils } \\
\text { choisissent une vignette et doivent produire un texte qui décrit le } \\
\text { quotidien de la personne choisie. } \\
\text { Objectif communicatif : Ecrire un article (pour un blog, journal) } \\
\text { qui décrit le quotidien de jeunes adolescents dans d'autres pays. } \\
\text { - Evaluation intermédiaire de la production écrite d'un récit } \\
\text { chronologique. }\end{array}$ & 305 \\
\hline
\end{tabular}

\subsection{Opérationnaliser de nouveaux savoirs dans des séquences d'enseignement}

\subsubsection{Place et statut des constituants à travers la progression informationnelle}

En amont des tâches wochenplan et kino, il est nécessaire d'avoir mis en place un certain nombre de prérequis. Les apprenants ont notamment assimilé le lexique relatif à différentes activités sous forme de syntagmes nominaux (Fußball - [le foot]) ou verbaux (Fußball spielen - [jouer au foot]) ; ils sont capables d'indiquer les jours de la semaine, des horaires et de formuler des questions. Les activités qui préparent à la tâche consistent tout d'abord à repérer des attaques différentes de l'énoncé dans un input oral indiquant quand ont lieu les clubs proposés à l'école. Tantôt l'énoncé s'ouvre sur une indication temporelle, tantôt c'est l'activité qui est donnée en premier. Ces choix ne sont pas arbitraires et les apprenants les associent très vite à deux types de question qui conditionnent deux linéarisations distinctes : dans un cas une attaque de type S-V-ADV, dans l'autre une construction au sein de laquelle se réalise l'Inversion ADV-V-S.

Suite à ce premier repérage et à de premières explications, différentes micro-tâches sont effectuées de manière à faire manipuler cette alternance de choix à l'ouverture et à lui donner une motivation fonctionnelle : pourquoi une information doit-elle de préférence apparaitre en fin d'énoncé ? Des activités qui associent le faire au dire, comme par exemple compléter un tableau vierge à partir d'informations entendues, permettent de saisir cette nécessité. Ces activités semi-guidées laissent ensuite la place à des activités plus personnelles : faire choisir des activités de clubs au sein de l'école et décrire ses choix, organiser des jeux de rôle avec des emplois du temps d'élèves allemands ou imaginer l'emploi du temps idéal lors d'une semaine de vacances, entre autres exemples.

L'enjeu ici réside dans le fait de faire saisir aux apprenants cette variation dans l'organisation des constituants et de leur faire manipuler les variations entre les deux options de linéarisation en fonction de la progression informationnelle : attaque par une expression temporelle $v s$ ouverture par le sujet. Le choix de l'élément préverbal ne varie en effet pas sans raison: une ouverture qui pose d'abord l'indication temporelle permet à l'information attendue (l'activité) d'être énoncée en dernier et d'être ainsi retenue pour être inscrite aux horaires repérés dans le planning : Was machst du am Donnerstag? [Qu'est-ce que tu fais le jeudi ?]. A l'inverse, la question Um wie viel Uhr ist Computerkurs?[Quand

${ }^{13}$ Le document support, extrait du manuel Team Deutsch NEU 1, est reproduit plus loin. 
est-ce que c'est le cours d'informatique ?] ne sera utile que pour vérifier que l'activité a été reportée dans le bon créneau. Il s'agit d'illustrer dans un premier temps la pertinence de ce choix, en lien avec la tâche à réaliser, à savoir compléter un tableau vierge. Mais il est aussi important de sensibiliser les apprenants au fait que des formes semblables n'ont pas la même valeur informationnelle, selon qu'elles apparaissent dans le Vorfeld ou en fin d'énoncé. Ainsi, un schéma de type Adverbial temporel-Verbe-Sujet pose-t-il un cadre à l'énoncé, alors que le positionnement final de cette même expression temporelle marque sa qualité d'information nouvelle. A l'explication grammaticale positionnelle se substitue donc une description fondée sur la manière de distribuer l'information dans l'énoncé en fonction des attentes formulées dans la question.

\subsubsection{Le décalage à l'ouverture et construction textuelle}

Dans la tâche tagesablauf, les apprenants ont à décrire le déroulement d'une journée d'école en inscrivant la succession des événements dans un cadre chronologique approprié. Cette tâche est l'occasion d'aborder la construction spécifique du récit chronologique et d'explorer avec les apprenants la manière dont s'enchainent en allemand les différents intervalles mentionnés.

Pour que les apprenants soient en mesure d'investir le champ initial de manière conforme, Dalmas (2005) préconise un travail à partir d'exemples de textes authentiques. Le site Kaleidoskop ${ }^{14}$, dédié au partage de courts récits du quotidien, rassemble des productions postées par des locuteurs natifs sur différents thèmes, dont la description des activités routinières durant une journée. Différentes contributions de locuteurs natifs ont été utilisées comme input en début de séquence et ont permis le repérage, non seulement des formes utiles à l'établissement de la succession chronologique, mais également de la manière particulière dont la succession se réalise.

Si on observe la production postée ici par un jeune allemand ${ }^{15}$ pour décrire sa journée d'école, on constate que le récit est découpé en intervalles temporels successifs et que l'information se déploie à l'intérieur de cette succession d'intervalles.

(5) Hallo Nikolas, Am Montag morgen gehe ich in die Schule. Manchmal gehe ich gern in die Schule aber manchmal auch nicht. Um 10.20 Uhr ist dann große Pause. Die Pause dauert ungefähr $20 \mathrm{~min}$. Meistens spiele ich dort dann Fußball. Dort bekomme ich dann im Dreck eine dreckige Hose. Nach der Schule laufe ich nach Hause und Esse zum Beispiel Kartoffeln mit Fleisch. Danach mach ich sofort die Hausaufgaben am Esstisch. Nach den Hausaufgaben muss ich zur Ensembleprobe. Dort spielen wir dann auf der Posaune und andere auf der Tuba. Wenn wir fertig gespielt haben laufe ich mit der Posaune nach hause. Zu Hause Esse ich was und danach gehe ich zu Bett. Das war mein Montag der ich dir sagen will.

Viele Grüße Valentin

[Salut Nikolas,

\footnotetext{
${ }^{14}$ Il s'agit d'un site géré par Wolfgang Hieber et qui propose d'échanger en allemand à partir de contributions qui décrivent des événements ou des faits du quotidien : habitudes, rituels, fêtes (Alltagstelegramme) ou qui prennent position sur différents thèmes. Les lecteurs peuvent réagir et poster en commentaires des contributions sur le sujet choisi. Le site est accessible à l'adresse suivante : http://www.kaleidos.de/

${ }^{15}$ Cette contribution et les réponses qui y sont associées sont consultables en ligne à l'adresse: www.kaleidos.de/category/alltagstelegramme/mein-tag/. Le texte est reproduit tel qu'il apparait sur le site.
} 
Le lundi matin, je vais à l'école. Parfois j'aime bien aller à l'école mais parfois pas non plus. A $10 \mathrm{~h} 20$ c'est la récré. La récré dure environ $20 \mathrm{~min}$. La plupart du temps je joue pendant ce temps au foot. Là il m'arrive alors d'avoir un pantalon tout sale dans la saleté. Après l'école, je rentre à pied à la maison et je mange par exemple des pommes de terre et de la viande. Après ça je fais tout de suite mes devoirs sur la table à manger. Après les devoirs je dois aller aux répétitions de l'ensemble. Là-bas on joue alors du trombone et d'autres jouent du tuba. Quand nous avons fini de jouer, je rentre à la maison avec mon trombone. A la maison, je mange un truc et après ça je vais au lit. C'était mon lundi, que je veux (voulais) te dire (raconter). Amitiés Valentin]

En relevant les expressions à l'ouverture (éléments soulignés), on peut constater que chaque transition présente à l'ouverture se réfère à un élément de l'énoncé précédent qui lui sert de point de repère.

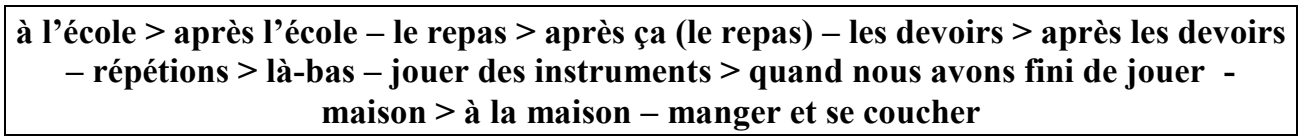

Fig. 2. Etablissement du décalage temporel dans le récit chronologique de Valentin.

Von Stutterheim parle de décalage (Verschiebung) pour caractériser ce mouvement particulier qui permet un déploiement de l'information dans la structure chronologique ainsi créée.

Lorsqu'un nouveau référent est introduit en établissant une relation explicite avec un référent déjà mentionné. C'est typiquement le cas dans les domaines conceptuels du temps et de l'espace, et notamment dans des expressions telles que «danach, davor » [avant ça / après ça] ou "darauf, dahinter » [là-dessus , là-derrière] ». L'usage de ces expressions permet dans le même temps de maintenir un référent (par le biais de l'élément anaphorique $d a(r)$ - ) (von Stutterheim, 1997, p. 63) ${ }^{16}$.

Sur le même thème, des contributions ont été postées en réponse au message de Valentin par des apprenants francophones. Plusieurs d'entre elles ont été postées un même jour, ce qui nous conduit à penser qu'il s'agit de productions effectuées dans le cadre d'un cours de langue. Confrontées au texte de Valentin, les réponses de ces apprenants francophones frappent par l'absence de structuration, qui transparait également dans la mise en page comme le montrent les exemples (6) et (7). Les énoncés produits se réduisent à des phrases isolées, ils sont certes corrects sur le plan syntaxique mais ne permettent pas à l'information de se déployer à l'intérieur d'une chronologie qui répond aux critères de textualité qui auraient pu être attendus. Il est dommage que les aspects textuels présents dans le document initial n'aient pas été exploités : c'est-à-dire que les schémas présents n'aient été ni repérés, ni n'aient fait l'objet d'explicitations. Ces différentes productions peuvent pourtant fournir aux apprenants l'occasion de mener une réflexion contrastive sur l'élaboration textuelle afin de dégager des critères généraux et spécifiques à la langue pour l'élaboration d'un texte. Au lieu de cela, des énoncés isolés sont produits et construits de manière arbitraire et mécanique. Même si les énoncés produits ci-dessous par L. commencent par un élément distinct du sujet ich [je], ce choix n'est pas nécessairement

\footnotetext{
${ }^{16}$ La citation est traduite par nos soins.
} 
motivé et la continuité chronologique que l'on pouvait observer dans le texte source n'est pas reproduite.

(6) Hallo

ich bin $O$.

ich wohne in Frankreich,

MEIN TAG:

Ich stehe um 7.00 auf

Ich frühstücke um 7.30

Ich gehe in die Kirche um 10.00

Ich sehe fern um 14.00 .

Ich besuche die Familie am Sonntag.

Ich schlafe um 21.00

Also Tschüss und bis Bald!

O.

(7) Am Montag

Ich bin L. Ich wohne in G. in Frankreich.

Um 7.00 Uhr stehe ich auf.

Um 7.10 Uhr frühstücke ich.

Um 7.15 Uhr nehme ich den Bus.

Um 7.45 Uhr habe ich Schule.

Um 10.00 Uhr mache ich Pause.

Um 12.00 Uhr esse ich zu Mittag.

Um 13.00 Uhr male ich.

Um 15. 00 Uhr schreibe ich.

Um 16. 00 Uhr trinke ich Kakao

Um 17. 00 Uhr gehe ich schlafen.

Also Tschüss und bis Bald!

$L$.
[Salut

je suis $\mathrm{O}$.

j'habite en France

MA JOURNEE:

Je me lève à $7 \mathrm{~h}$.

Je prends mon petit déjeuner à $7 \mathrm{~h}$ 30

Je vais à l'église à $10 \mathrm{~h}$.

Je regarde la télé à $14 \mathrm{~h}$

Je rends visite à la famille le dimanche.

Je dors à $21 \mathrm{~h}$.

Alors salut et à bientôt

O.]

[Le lundi

je suis L. et $\mathrm{j}$ 'habite en France.

A $7 \mathrm{~h}$ je me lève

A 7 h10 je prends mon petitdéjeuner

A 7 h15 je prends le bus.

A 7 h45 j'ai école.

A $10 \mathrm{~h}$ je fais une pause

A $12 \mathrm{~h}$. je mange le repas de midi

A 13 h. je peins

$114 \mathrm{~h}$. j'écris

A $16 \mathrm{~h}$. je bois un chocolat chaud

A $17 \mathrm{~h}$ je vais me coucher

Alors salut et à bientôt

L.] 
En amont de la réalisation de la tâche tagesablauf, les apprenants ont donc effectué des activités destinées à préparer un traitement cognitif adéquat de la tâche finale : des moyens langagiers spécifiques et des schémas d'usage ont été repérés au sein des contributions tirées du site Kaleidoskop, notamment les adverbes prépositionnels tels que dann et danach [ensuite, après ça], dont le fonctionnement anaphorique et la fonction de raccord ont été explicités. Les apprenants ont ensuite procédé à une restitution orale des textes ayant servi d'exemples, puis ont produit des textes personnels dans lesquels ils ont été invités à varier les formulations et à prêter attention à la construction globale de leur production écrite. La tâche finale tagesablauf a servi à évaluer la prise en compte de ces aspects.

Pour réaliser cette tâche, les apprenants disposent d'un input volontairement homogène. Il s'agit d'un extrait du manuel Team Deutsch 1 (Esterl, Körner, \& Einhorn, 2008, p. 58) reproduit dans le document 1 . Pour trois adolescents de pays différents, une liste d'activités quotidiennes est associée à des horaires.

Document 1. Document support fourni pour la tâche tagesablauf.

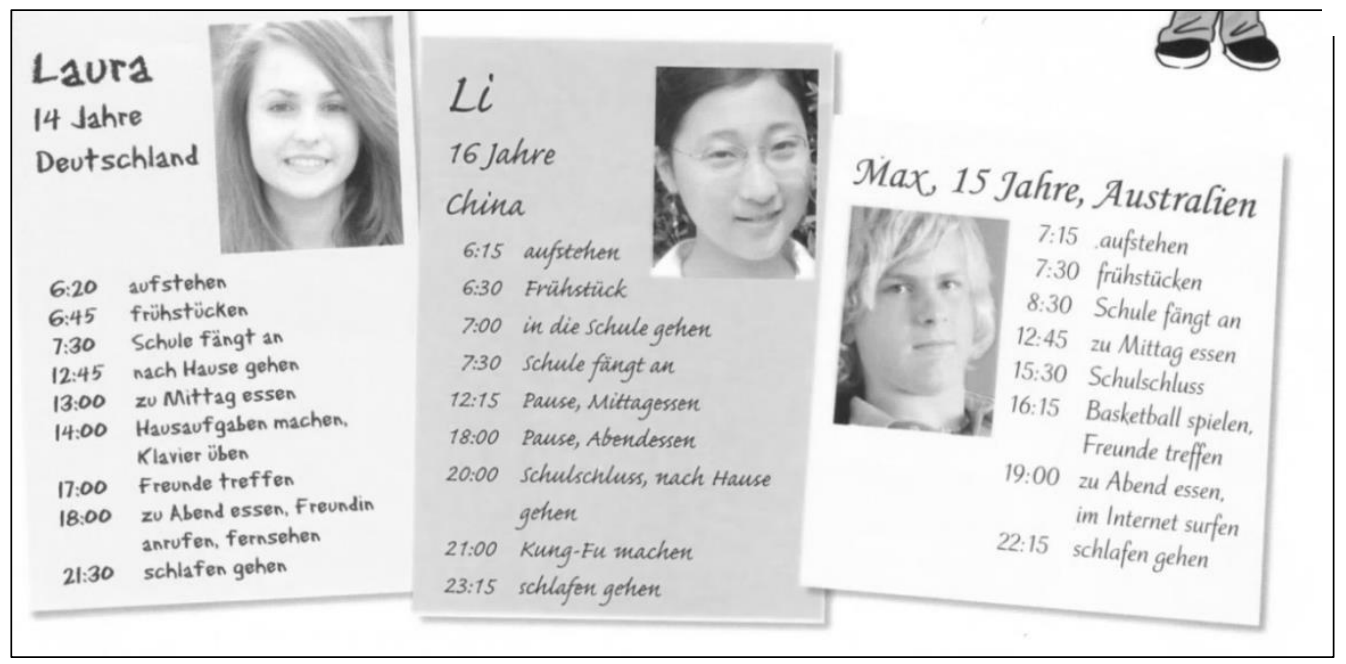

La tâche consiste à rédiger, à partir de ces informations, un article (pour un journal ou un blog interculturel) racontant le quotidien d'un adolescent vivant dans un autre pays. Il est attendu des apprenants qu'ils produisent un texte construit et réinvestissent des moyens langagiers découverts et manipulés en amont de manière à ce que leur production écrite se rapproche plus de celle de Valentin que des phrases postées sur le site Kaleidoskop par leurs homologues francophones.

\subsection{Traitement des données et analyse}

Une fois les tâches effectuées, les données recueillies ont été transcrites, et ce faisant des erreurs morphosyntaxiques qui n'affectaient pas l'ouverture ont été corrigées afin de faciliter la compréhension des énoncés (il s'agit des rajouts entre crochets en (8) et (9) par exemple).

(8) (NEI_PIE_wochenplan)

173. PIE: Am Mittwoch um eins / von eins [ein] Uhr bist [bis] 3 Uhr spielt ich / spiele ich Basket [Basketball] und um 5 Uhr habe ich Bibliothek.

174. NEI: Danke. Was [wann] machst du deine Hausaufgaben? 
(9) (JUL_tagesablauf)

67. Um fünf Uhr trefft [trifft] sie [sich] mit ihr [en] Freunde [n] und eine Stunde nach [danach]isst sie zu Abend, sie ruft Freundin [nen] an und sie siehst [sieht] fern. Um halb 10 geht sie schlafen. Das ist Laura's [Lauras] Tag!

Les transcriptions ont été ensuite segmentées à l'aide de l'outil Partitur Editor ${ }^{17}$ de EXMARaLDA (Schmidt \& Wörner, 2009), de manière à annoter les constituants occupant le Vorfeld à plusieurs niveaux : syntaxique, informationnel et textuel.

Ces annotations ont livré des résultats quantitatifs sur le taux de réalisation correcte et incorrecte du Vorfeld, à savoir sur la réussite du positionnement verbal second, qui a été rapportée aux catégories de constituants choisis pour l'ouverture. Sur le plan informationnel et textuel, elles ont mis en évidence la pertinence des choix faits à l'ouverture en fonction des énoncés adjacents ou de la structure globale du texte. Les données chiffrées ont par conséquent toujours été confrontées à des réalisations particulières, considérées dans leur contexte d'insertion. Les résultats obtenus, ainsi que l'observation des productions, livrent des informations quant à la manière dont les apprenants réinvestissent des formes langagières dans une production personnelle suite aux différentes séquences d'enseignement présentées plus haut. Ils permettent de déterminer si certaines procédures paraissent plus accessibles, lesquelles au contraire peuvent s'avérer plus exigeantes en termes de traitement dans une situation de production moins guidée.

\section{Résultats}

\subsection{La gestion interactive de la progression informationnelle}

La tâche recueillie wochenplan se déroule selon un schéma interactif simple : chaque tour de parole se décompose en une question relative aux activités et une réponse apportant l'information demandée. La réalisation de la tâche s'accompagne de la confection d'un planning qui contraint le type d'informations à recueillir et les questions pouvant être posées. Deux linéarisations distinctes attribuent un statut différent à l'élément temporel et ont fait l'objet de manipulation et d'explications en amont. Il est ainsi possible soit de demander quelles activités se déroulent à différents moments (Welche sind deine Aktivitäten in der Woche? Was hast /machst du am Montag? [Qu'est-ce que tu fais comme activités dans la semaine ? Tu as / fais quoi le lundi ?]), soit de se renseigner sur le jour et l'heure de différentes activités (Wann ist Fußball? [Le foot, c'est quand?]). Ces deux types de questions déterminent l'organisation des constituants dans l'énoncé selon la distribution des rôles informationnels de topique et de focus et sont bien présentes dans les productions recueillies.

(10) (ULY_ART_wochenplan)

251. ART: $\overline{O K}$. Und am Donnerstag / was machst du am

Donnerstag?

[OK. Et le jeudi / qu'est-ce que tu fais le jeudi?]

252. ULY: Am Donnerstag habe ich Computerkurs. (ja) Und um 4 Uhr spiele ich Flöte.

[Le jeudi j'ai cours d'informatique. Et à 4 heures je joue de la flûte.]

253. ART: Computerkurs? Um wieviel Uhr ist Computerkurs?

\footnotetext{
${ }^{17}$ Documentation, logiciels et tutoriels sont disponibles à l'adresse suivante : http://www.exmaralda.org/
} 
[Cours d'informatique? A quelle heure c'est le cours d'informatique ?]

254. ULY: Computerkurs ist am Donnerstag um 2 Uhr.

[Le cours d'informatique est le jeudi à 2 heures.]

On peut, dans un premier temps, faire remarquer que dans de nombreuses productions recueillies, les choix d'organisation informationnelle sont pertinents ${ }^{18}$ et correspondent à l'organisation informationnelle induite par la question. L'élément donné (le topique) sur lequel porte la question est repris à l'ouverture et l'information attendue (le focus) apparait bien en fin d'énoncé.

(11) (MARN_CLAR_wochenplan) 156. CLAR: Wann ist / Entschuldigung / was machst du am Mittwoch?

[C'est quand / pardon / qu'est-ce que tu fais le mercredi ?]

157. MARN: Am Mittwoch um 2 Uhr habe ich Schulorchester und am 3 Uhr / um 3 Uhr spiele ich Flöte und um 17 Uhr habe ich Tanzen und du?

[Mercredi à deux heures $\mathrm{j}$ 'ai orchestre et à trois heures je joue de la flûte et à 5 heures j'ai danse et toi ?]

158. CLAR : Wann ist Flöte?

[C'est quand la flûte ?]

159. MARN: Flöte ist am Mittwoch um 3 Uhr.

[La flûte, c'est le mercredi à trois heures.]

La séquence (11) met en évidence le changement de statut de l'information temporelle entre les énoncés (157) et (159). Si les formes (catégorie et fonction syntaxiques) sont similaires, les éléments temporels constituent une information nouvelle en (159) qui correspond au focus, c'est-à-dire à un contenu informationnel apporté en réponse à la question Wann?[Quand ?]) ; alors qu'en (157), l'intervalle temporel représente une donnée informationnelle préalablement donnée par le locuteur et qui fait figure de cadre dans lequel est circonscrite l'information demandée par la question Was? [Quoi / Que ?]. Ces choix de linéarisation semblent bien conscients chez certains apprenants, comme en témoigne l'échange (12) entre TEO et MARC dans lequel ce dernier n'hésite pas à souffler ${ }^{19}$ à son partenaire l'option qui lui semble le mieux convenir.

(12) (TEO_MARC_wochenplan)

213. TEO: Was machst du am Freitag?

[Tu fais quoi le vendredi ?]

214. MARC: Am Freitag um 3 Uhr spiele ich Tischtennis und am Freitag um 4 Uhr habe ich Schokofondüe bei Yohan, meine best Freundin [meinem besten Freund]. Und du am Montag, spiele / spielst du Handball am Montag?

[Vendredi à 3 heures, je joue au ping-pong et vendredi à 4 heures j'ai une fondue au chocolat chez Yohan, mon meilleur copain. Et toi le lundi, est-ce que tu joues au hand le lundi ?]

215. TEO: Nein, am (Handball) / Handball (ist) ist am Mittwoch um 4 Uhr.

\footnotetext{
${ }^{18}$ Les résultats indiquent un taux d'inadéquation plus grand pour la tâche kino $(22,1 \%)$ que pour la tâche wochenplan $(4,9 \%)$.

${ }^{19}$ Les rajouts entre parenthèses en (215) indiquent une intervention du partenaire dans la production du locuteur.
} 
[Non, lun (le hand) / le hand (c'est) c'est le mercredi à 4 heures.]

\section{2 Les réalisations du positionnement verbal second}

Pour les deux tâches wochenplan et kino, les réalisations correctes du Vorfeld, c'est-à-dire le positionnement verbal second correct, excèdent $80 \%$ sur l'ensemble des énoncés pris en compte. Pourtant, l'analyse catégorielle des constituants en position préverbale (partie droite de la Fig.3) montre dans ces cas de réussite une occupation privilégiée de l'ouverture par des éléments distincts du sujet (ici principalement des adverbiaux temporels plus ou moins complexes); il est intéressant de noter qu'en dépit de cette préférence, qui nécessite de réaliser l'Inversion, le placement verbal s'avère majoritairement correct chez un grand nombre d'apprenants.

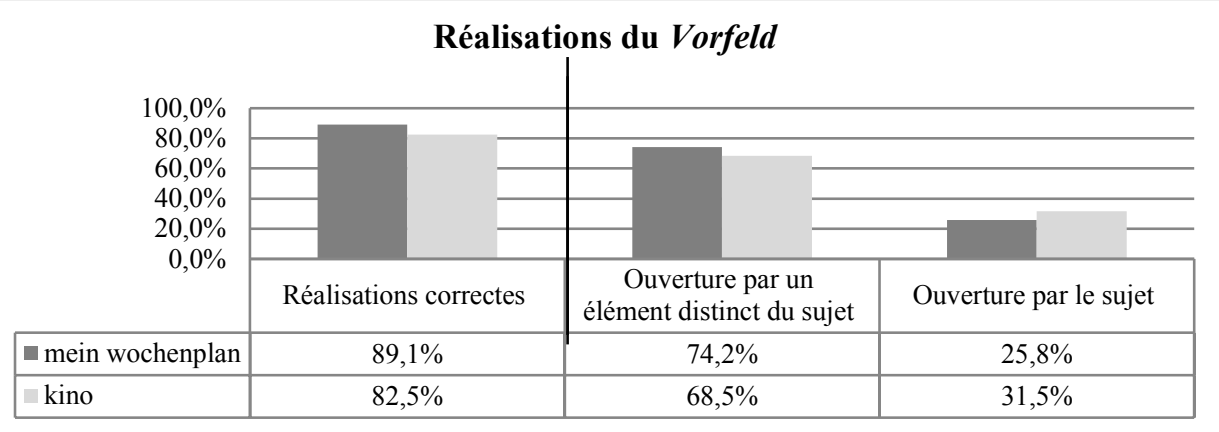

Fig. 3. Répartition des réalisations du Vorfeld dans les tâches wochenplan et kino.

Si l'on va plus loin dans l'observation et que l'on rapporte les réalisations correctes aux bases verbales employées (partie gauche de la Fig.4), on constate que les apprenants ont recours dans la tâche wochenplan à une plus grande variété de bases verbales, notamment spielen et machen [jouer, faire] pour les plus fréquentes à côté de sein et haben [être (c'est), avoir (il y a)], alors que dans la tâche kino, ils privilégient des constructions formées à partir des seules bases verbales sein et haben. Des énoncés de type Am Montag um 2 ist / habe ich Theater [Le lundi à 2 heures il y a / j'ai théâtre] sont ainsi privilégiés par rapport à Am Montag um 2 spiele ich Theater [Le lundi à 2 heures je joue au théâtre.] La variété que l'on peut observer dans les exemples (13) et (14) ne se retrouve plus dans la tâche kino.

Les erreurs relatives au positionnement verbal second (partie droite dans la Fig.4) sont quantitativement comparables dans les deux tâches, mais rapportées à la variété des bases verbales, on constate une moindre réussite dans la réalisation de l'Inversion dans la tâche kino qui laisse supposer un recours à un fonctionnement calqué sur les structures de la L1.

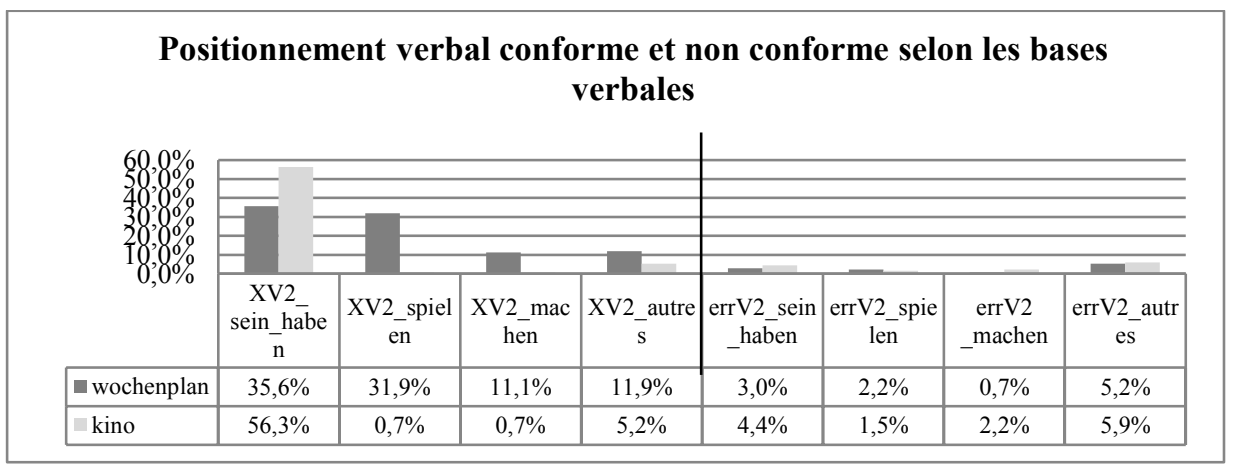


Fig. 4. Distribution du positionnement verbal second dans les tâches wochenplan et kino selon les verbes utilisés.

(13) (IFI_MAU_wochenplan)

89. IFI: Und du was machst du am Freitag?

[Et le vendredi, qu'est-ce que tu fais?]

90. MAU: Am Freitag um 2 Uhr habe ich Musiktheorie, am Freitag um 3 Uhr spiele ich Theater und um 4 Uhr nein und um 5 Uhr spiele ich Fußball und um 6 Uhr habe ich Naturprojekt.

[Le vendredi à 2 heures j'ai solfège, le vendredi à 3 heures je fais du théâtre et à 5 heures je joue au foot et à 6 heures j'ai le projet nature.]

91. IFI: Gut, wann gehst du schwimmen?

[Bien, et quand est-ce que tu vas nager ?]

92. MAU: Ich spiele [gehe] schwimmen am Montag um 4 Uhr. [Je joue (je vais) nager le lundi à 4 heures.]

(14) (ELE_CLAI_wochenplan)

29. $\overline{C L A I}$ : $\bar{A}$ m Montag um 3 mache ich Fossilienclub und um 5 mache ich Ballett mit Elke. Und du, was machst $d u$ am Montag?

[Le lundi à 3 heures je fais club fossile et à 5 heures je fais de la danse classique avec Elke. Et toi, qu'est-ce que tu fais le lundi ?]

30. ELE: Am Montag um 2 tanze ich, um 4 spiele ich Klavier und um 5 Hausauf / mache ich Hausaufgaben.

[Le lundi à 2 heures je danse, à 4 heures je joue du piano et à 5 heures je fais mes devoirs.]

La tâche wochenplan s'assimile à une tâche formative, mettant en jeu un schéma de question/réponse prévisible qui rend possible un contrôle et par delà une réussite du positionnement verbal malgré l'utilisation d'occurrences verbales, certes limitées mais cependant variées. La gestion de la tâche kino, dont le déroulement est quant à lui moins répétitif, semble en revanche requérir plus de ressources attentionnelles. Cette nécessaire mobilisation attentionnelle au service de la gestion d'une interaction moins mécanique peut expliquer les erreurs et le besoin de recourir à des préférences de la L1 ou à des constructions en L2 plus directement accessibles, telles que les associations formées à partir de la base verbale sein [c'est / il y a]: Am Montag ist... [Le lundi, c'est / il y a...] ou à partir du bloc habe ich [j'ai]: Am Montag habe ich ...[Le lundi, j'ai] comme dans les exemples (15) et (16).

(15) (ANT_YOH_kino)

9. ANT: Am Mittwoch um 2 Uhr ist Computerkurs und am Mittwoch um 4 Uhr ist Hausaufgaben. Und du, was machst du am Dienstag?

[Le mercredi à 2 heures il y a cours d'informatique et le mercredi à 4 heures il y a les devoirs. Et toi, qu'est-ce que tu fais mardi ?]

(16) (ROM_EVA_kino) 


\section{ROM: Am Montag um 2 Uhr ist Theater-AG und am Montag um vier ist Schwimmen. Und $d u$ ? \\ [Le lundi à 2 heures c'est club théâtre et le lundi à 4 heures c'est natation. Et toi ?] \\ 52. EVA: Am Montag mach / habe ich / am Montag habe ich Street-dance [es] ist 3 Uhr und Hausaufgaben ist [um] 5 Uhr. Es geht nicht am Montag. \\ [Lundi, j'ai club Street Dance est à 3 heures et les devoirs, c'est 5 heures. Ça ne va pas lundi.]}

On constate par ailleurs que certains groupes verbaux, initialement formés avec spielen ou machen [jouer, faire], sont recomposés à partir de haben : Hausaufgaben haben [avoir les devoirs] vs Hausaufgaben machen [faire les devoirs]. Cette tendance est encore plus frappante dans l'exemple (17) avec l'énoncé (202): Schwimmen haben [avoir natation] vs schwimmen gehen [aller nager] ou encore en (18) dans les énoncés (117) et (118) avec Sport haben [avoir sport] vs Tennis spielen / Fußball spielen [jouer au tennis / au foot].

(17) (PIE_NEIG_kino)

200. NEI: Hast du Zeit am Montag?

[Est-ce que tu as du temps lundi ?]

201. PIE: Nein, am / am 3 Uhr / um 3 Uhr habe ich Street

Dance und am[um] 5 Uhr habe ich Hausaufgaben. Und du, hast du Zeit am Montag?

[Non, à 3 heures j'ai Street Dance et à 5 heures j'ai les devoirs.

Et toi, est-ce que tu as le temps lundi ?]

202. NEI: Ich / Nein, es geht nicht. Am / am / um 2 Uhr habe

ich Theater-AG und um 4 Uhr habe ich Schwimmen. Und am

Dienstag, hast du Zeit?

[Non, ça ne va pas. A deux heures, j'ai club théâtre et à 4 heures j'ai natation. Et mardi, est-ce que tu as le temps ?]

(18) (TEO_MARC_kino)

116. TEO: (...) Und am / um 17 Uhr habe ich Hausaufgaben.

[Et à 5 heures j'ai les devoirs.]

117. MARC: Ah OK. Unmöglich. Am Donnerstag habe ich Sport: Tennis und du?

[Ah, OK, impossible. Jeudi j'ai sport : tennis et toi ?]

118. TEO: Am Montag um 16 Uhr habe ich Sport: Fußball.

[Lundi à 4 heures j'ai sport : foot.]

Cette recomposition à partir de «ist» [c'est / il y a] ou de «habe ich » [j'ai] permet d'utiliser des groupes nominaux (Fußball, Sport, Hausaufgaben [le foot, le sport, les devoirs]) à la place des groupes verbaux, c'est-à-dire des constructions qui nécessitent le rappel et l'intégration d'un seul item lexical au sein d'un bloc et non le réagencement de plusieurs constituants. De telles constructions ont été les plus fréquemment utilisées dans les activités préparatoires et on peut considérer que leur rappel se trouve facilité par leur degré d'automatisation. L'utilisation de tels blocs évite le recours à une élaboration plus analytique et nécessairement moins accessible, car moins automatisée, telle que : $\mathrm{Am}$ Montag + spiele $+i c h[$ Le lundi + joue $+\mathrm{je}]$. Un traitement nominal se substitue ainsi à des opérations syntaxiques plus complexes et nécessairement plus couteuses sur le plan attentionnel. 


$$
\begin{gathered}
\text { traitement nominal : ADV ist + NP ou ADV habe ich + NP } \\
\qquad v s \\
\text { traitement syntaxique : } \mathbf{A D V}+\mathbf{V}+\mathbf{S}+\mathbf{N P}
\end{gathered}
$$

Fig. 5. Substitution d'un traitement nominal à un traitement syntaxique.

Dans les deux tâches, le schéma interactionnel et la progression informationnelle ont favorisé la répétition de très nombreuses séquences dans lesquelles un élément, mentionné dans la question, est repris comme amorce de l'énoncé suivant. Il est particulièrement intéressant d'observer que l'entrainement à cette reprise systématique a pu faire émerger, dans un usage spontané, une structure spécifique de la L2, à savoir une construction de type Objet-Verbe-Sujet caractérisée par le positionnement d'un complément à l'accusatif dans le Vorfeld, comme dans l'énoncé (140) de l'exemple (19).

(19) (MEL_MAR_wochenplan)

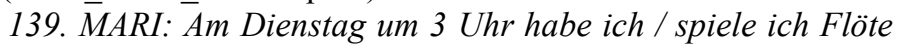
und am 5 habe ich Schulorchester. Wann ist / wann ist Florettfechten?

[Mardi à 3 heures j'ai / je joue de la flûte et à 5 heures j'ai orchestre. C'est quand le fleuret?]

\section{MEL: Florettfechten spiele [mache] ich am Mittwoch um 4 Uhr. Wann ist Klavier? \\ [Le fleuret, j'en fais le mercredi à 4 heures. Le piano, c'est quand ?]}

L'énoncé est non seulement pertinent sur le plan de la progression informationnelle - il y a bien reprise du connu en attaque et apport informationnel par la suite ; il est également correct d'un point de vue syntaxique, malgré la spécificité de cette construction qui, en français, serait rendue par une thématisation, telle qu'elle est exprimée dans la traduction correspondante.

\subsection{De la phrase isolée à une construction textuelle spécifique}

La consigne donnée pour la tâche tagesablauf invite les apprenants à décrire le déroulement de la journée d'un adolescent. Dans la majorité des productions recueillies $(67,4 \%)$, le choix est fait d'organiser la trame du récit à partir des intervalles temporels plutôt que d'adopter une perspective centrée sur le protagoniste (27,6\%). Cette perspective correspond en effet à la question sous-jacente, qui est de savoir quelles activités s'échelonnent dans la journée et non d'apporter des informations sur une personne particulière. La confrontation des exemples (20) et (21) illustre deux options distinctes dans les choix d'organisation textuelle et dans le déploiement informationnel au sein des énoncés et du texte produit. Le texte de MARI se présente en effet comme une juxtaposition d'informations sur le personnage choisi, alors que IFI préfère présenter le déroulement de la journée de Laura dans une chronologie d'intervalles.

(20) (MARI tagesablauf)

89. Morgens stehe [steht] sie um zwanzig nach sechs auf und dann frühstückt sie, es ist viertel vor sieben. Sie geht in die Schule um halb acht. Der Unterricht enden [endet] um Viertel vor eins und sie isst um eins. Nach dem Essen macht sie seine [ihre] Hausaufgagen und übt Klavier. Sie kann Freunde treffen um fünf. Sie isst um sechs und sie ruft eine Freundin an und sehe [sieht] sich fern. Sie geht schlafen um halb zehn. 
[Le matin, elle se lève à six heures vingt et ensuite elle prend son petit-déjeuner, il est sept heures moins le quart. Elle va à l'école à sept heures et demie. Les cours se terminent à une heure moins le quart et elle mange à une heure. Après manger, elle fait ses devoirs et travaille son piano. Elle peut retrouver des amis à cinq heures. Elle mange à six heures et elle téléphone à une amie et regarde la télé. Elle va se coucher à neuf heures et demie.]

(21) (IFI_tagesablauf) 55. Einen [Ein] deutsch [er] Tagesablauf

Hallo! Heute beschreibe ich Lauras Tagesablauf. Also, Laura steht um 6:20 auf und zwanzig Minuten danach frühstückt sie. Um halb acht fangt [fängt] die Schule an und um eins isst sie zu Mittag. Eine Stunde danach macht sie Hausaufgaben und spielt sie Klavier bis um fünf, da trefft [trifft] sie Freunde aber um sechs soll sie zu Hause sein. Da isst sie nämlich zu Abend, dann ruft sie Freundin[nen] an und sieht sie fern vor schlafen gehen um halb zehn.

[Le déroulement d'une journée en Allemagne

Salut ! Aujourd'hui je décris la journée de Laura. Donc, Laura se lève à $6 \mathrm{~h} 20$ et 20 minutes après elle prend son petit déjeuner. A 7 heures et demi, l'école commence et à une heure elle a le repas de midi. Une heure plus tard elle fait ses devoirs et elle joue du piano jusqu'à 5 heures. Là elle retrouve des amis mais à 6 heures elle doit être à la maison. C'est à cette heure en effet qu'elle mange le repas du soir, ensuite elle téléphone à des amis et elle regarde la télé avant d'aller dormir à 9 heures et demi.]

Le texte produit par IFI n'est pas sans rappeler celui de Valentin cité plus haut. Il y est instauré non seulement une continuité temporelle, dans la mesure où la borne d'un segment sert de repère pour le segment suivant, mais également une continuité propositionnelle à travers les nombreuses reprises anaphoriques dont elle fait usage. Il est également pertinent que le décalage temporel ne se mette en place qu'une fois le topique Laura introduit dans la première phrase.

Ce qui est frappant, c'est la très grande variété des formes produites pour établir la chronologie temporelle et le décalage que l'on peut recenser dans les productions. Par rapport au document support fourni pour la tâche (Doc. 1), aucun apprenant ne choisit la facilité en se contentant de répéter les horaires donnés. Au lieu de cela, tous créent une trame originale en utilisant des formes connues, en les recomposant ou encore en fabriquant des tournures idiosyncrasiques, comme en témoignent les occurrences relevées dans le tableau 5 .

Tableau 5. Variété des expressions temporelles pour la délimitation d'intervalles successifs. 


\begin{tabular}{|c|c|}
\hline Groupes prépositionnels & $\begin{array}{l}\text { um halb acht [à sept heures et demie] } \\
\text { von siebzehn Uhr bis etwa achtzehn Uhr [de } 17 \text { jusqu'à } \\
\text { environ } 18 \text { heures] } \\
\text { nach dem Frühstück [après le petit-déjeuner] } \\
\text { nach dem Essen [après le repas] } \\
\text { nach den Hausaufgaben [après les devoirs] } \\
\text { nach dem Basketball spielen [après avoir joué au basket] } \\
\text { nach [dem] }{ }^{20} \text { Klavier üben [après les exercices au piano] } \\
\text { nach der [dem] Unterricht [après les cours] } \\
\text { nach der [dem] Schulschluss [après la fin des cours] }\end{array}$ \\
\hline Groupes adverbiaux & zuerst [d'abord] , dann [ensuite] , morgens [le matin] \\
\hline $\begin{array}{l}\text { Groupes nominaux avec } \\
\text { expansion }\end{array}$ & $\begin{array}{l}\text { eine Stunde nach dem Essen [une heure après manger] } \\
\text { etwa } 45 \text { Minuten später [environ } 45 \text { minutes plus tard] } \\
\text { ungefähr eine halbe Stunde später [à peu près une demi- } \\
\text { heure plus tard] } \\
\text { [Eine]Viertelstunde später [un quart d'heure plus tard] }\end{array}$ \\
\hline Subordonnées & $\begin{array}{l}\text { wenn die Schule aus ist, [quand l'école est finie] } \\
\text { before [Bevor] sie ins Bett geht [avant qu'elle aille se } \\
\text { coucher] }\end{array}$ \\
\hline Expressions complexes & $\begin{array}{l}\text { dann, um sechs Uhr [ensuite à six heures] } \\
\text { nach dem Abendessen, wenn sie ihre Freundin an nicht ruft } \\
\text { [nicht anruft] [après le repas, si elle ne téléphone pas à ses } \\
\text { amies] } \\
\text { erst dann [c'est seulement ensuite que] }\end{array}$ \\
\hline Formes idiosyncrasiques & $\begin{array}{l}\text { nach ihre Freunde treffen [après avoir retrouvé ses amis] } \\
\text { vor dem Freunde treffe [avant de retrouver les amis] } \\
\text { drei Stunden früher [vor dem] schlafen gehen [trois heures } \\
\text { avant d'aller se coucher] } \\
\text { nach [danach] [après (adverbial)] } \\
\text { eine Stunde nach [danach] [une heure après (adverbial)] }\end{array}$ \\
\hline
\end{tabular}

Ce qui peut également paraitre surprenant est que la proportion de réalisations correctes du Vorfeld est assez importante malgré la complexité des constituants initiaux et la variété des formes verbales employées, comme le montrent les exemples (22) à (24) et les résultats chiffrés du graphique de la Fig. 6.

(22) (CLAR_tagesablauf)

22. Wenn die Schule zu Ende ist, geht sie nach Hause und ein

[e] Viertelstunde später isst sie zu Mittag.

[Quand l'école est finie, elle rentre à la maison et un quart d'heure plus tard elle mange.]

(23) (EMA_tagesablauf)

37. (...) um sechs Uhr nach dem zu Abend essen [Abendessen] ruft sie seine [ihre] Freundin [an].

$[(\ldots)$ à six heures après le repas du soir, elle téléphone à son amie.]

(24) (NEI_tagesabalauf)

\footnotetext{
${ }^{20}$ Les termes en allemand entre crochets indiquent les corrections que nous avons apportées. A noter que les formes idiosyncrasiques n'ont pas été rectifiées quand la forme correcte nécessite la reformulation d'une tournure prépositionnelle en une subordonnée.
} 


\section{1. (...) nach dem Abendessen wenn sie ihre Freundin an \\ nicht ruft [nicht anruft], sieht sie fern. \\ $[(\ldots)$ après le repas du soir, si elle n'appelle pas son amie, elle regarde la télé.]}

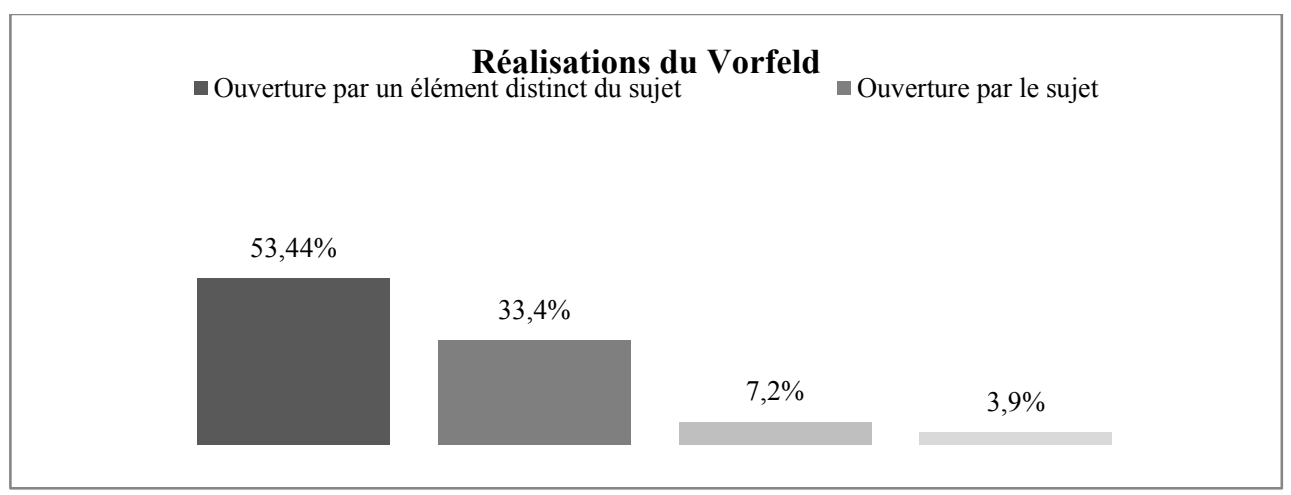

Fig. 6. Différentes réalisations du Vorfeld dans la tâche tagesablauf.

On peut supposer que la modalité écrite de la tâche favorise un positionnement verbal second correct, qui peut le cas échéant être contrôlé a posteriori. Il se peut aussi que la répétition d'associations Verbe-Sujet lors de la réalisation des tâches antérieures influence durablement l'ordre d'apparition du complexe V-S et conduise à des erreurs de surgénéralisation. Les cas de non-occupation du Vorfeld relevés (3,9\%) concernent principalement les énoncés coordonnés avec und ou oder [et, ou] et dans lesquels le sujet apparait de manière non conforme en position postverbale et vont selon nous dans le sens de cette interprétation.

(25) (IFI_tagesablauf)

58. Eine Stunde danach macht sie Hausaufgaben und spielt sie

Klavier bis um fünf.

[Une heure après, elle fait ses devoirs et joue elle du piano jusqu'à cinq heures.]

(26) (LOU_tagesbalauf)

75. Danach isst sie zu Abend und ruft sie seine [ihre] Freundin an oder sieht sie fern. Um 21:30 geht sie ins Bett.

[Après ça, elle mange le repas du soir et téléphone elle à sa copine ou regarde elle la télé. A $21 \mathrm{~h} 30$ elle va se coucher.]

La succession des intervalles temporels à l'ouverture fait partie de la construction textuelle spécifique dont la mise en place est visée ici, mais un deuxième aspect ressort également des observations menées, à savoir l'usage des éléments cohésifs qui participent également de la textualité en allemand. En effet, les expressions temporelles en position préverbale établissent un lien avec l'énoncé précédent en se référant à un contenu préalablement introduit. Dans le cas de später [plus tard], la reprise anaphorique est implicite (le point de référence précédent demeure activé sans être repris), elle est en revanche explicite avec des expressions telles que da, dann et danach [là, ensuite, après ça] dans lesquelles l'élément anaphorique $d a$ matérialise la portion d'espace - temporel ou local - préalablement construit (Marschall, 2012). 


\section{Discussion}

\subsection{Reprise, mémorisation et réalisation de structures syntaxiques spécifiques}

Plusieurs remarques importantes peuvent être formulées au vu des résultats relatifs aux tâches interactives wochenplan et kino. Ceux-ci livrent des pistes pour la mise en place d'activités susceptibles d'accompagner les mécanismes d'appropriation chez des apprenants en tout début d'apprentissage.

Le travail mené avec les apprenants sur le statut informationnel des constituants (donné, connu vs nouveau) permet tout d'abord de poser la question de la pertinence des catégorisations grammaticales traditionnelles. Positionnée à l'ouverture ou en fin d'énoncé, une expression adverbiale temporelle a certes la même forme mais pas le même statut et la catégorie «complément de temps» ne permet pas de distinguer ces valeurs informationnelles différentes, essentielles pour la réception des messages. La capacité à apprécier le statut informationnel des constituants nous semble, plus encore que des règles abstraites, devoir être introduite dans l'apprentissage afin de mettre en place des associations formes-fonctions qui soient ancrées dans un contexte communicatif qui les rende pertinentes.

Il apparait ensuite qu'en début d'apprentissage, un fonctionnement mémoriel et l'usage répété de constructions prototypiques, telles que celles employées pour décrire ces activités de la semaine et de type ADV-V-S, contribuent à rendre routinières des attaques de l'énoncé par un élément distinct du sujet tout en favorisant la fixation mémorielle des associations Verbe-Sujet. Une majorité d'apprenants n'a pas eu de difficulté à gérer la distribution de l'information en faisant alterner attaques cadratives et sujet (mention de l'activité) à l'ouverture; la plupart des énoncés produits s'avèrent corrects sur le plan syntaxique dans la mesure où le placement verbal second et l'Inversion sont réalisés quand un adverbial occupe la position préverbale. Il parait difficile de rapporter cette réussite au fait que les apprenants auraient déjà atteint le stade développement correspondant à une telle réalisation (stade $x+3$ : Inversion). Les résultats montrent en effet que la réussite est moindre avec des bases verbales moins fréquentes et lorsque le schéma interactif s'avère moins prévisible. Selon nous, la correction syntaxique observée est liée en partie au format de la tâche mais aussi à la disponibilité de certaines associations de type Adverbial+ist+SN ou encore Verbe-Sujet, notamment habe ich voire spiele ich) dont l'usage repéré et répété dans les activités préalables, et durant la tâche elle-même, favorise la mémorisation et le rappel.

La comparaison des deux tâches wochenplan et kino montre cependant qu'il semble raisonnable de ne pas viser dès le début la réalisation de V2 avec une trop grande variété de bases verbales, comme les enseignants peuvent avoir tendance à le faire une fois abordée la règle du positionnement verbal second. Durant la tâche formative (wochenplan), de courtes séquences en question / réponse portant sur un nombre relativement limité d'occurrences verbales ont fait l'objet d'un usage répété. La fréquence d'emploi, la répétition soutenue par l'interaction ainsi que le guidage opéré durant les phases préparatoires contribuent à fixer en mémoire un certain nombre de constructions de type Adverbial-Verbe-Sujet, ainsi que des associations Verbe-Sujet qui seront rappelées de manière privilégiée dans un contexte similaire. Les associations les plus fréquentes (habe ich / spiele ich ou ist), puis des bases verbales qui attestent également d'une assez grande fréquence d'emploi (machen, gehen), peuvent par conséquent constituer un premier palier avant l'introduction de verbes plus diversifiés.

Le caractère interactif des échanges s'avère également être ici un dispositif de soutien à l'expression. Le fait d'effectuer la tâche en binôme permet non seulement à l'interlocuteur 
d'être attentif à l'input fourni par le partenaire et, le cas échéant, d'ajuster sa propre production en fonction de l'input reçu. La reprise quasi-systématisée d'un élément temporel à l'ouverture, quand celui-ci est donné dans la question, facilite le positionnement frontal de cet élément et le choix d'une attaque cadrative. De la même façon, c'est par le biais de ce schéma récurrent qu'une apprenante (énoncé 140 dans l'exemple (19)) parvient à produire spontanément, dans l'usage, une construction spécifique de l'allemand avec un positionnement préverbal d'un complément à l'accusatif (ordre OVS). Dalmas fait remarquer à ce sujet :

Si un élément thématique, identifié syntaxiquement comme complément est positionné dans le Vorfeld, sa topicalisation est alors interprétée à tort lors de la réception du texte comme mise en valeur. Et à la suite de cela, en production, des compléments sont rarement, voire trop rarement topicalisés ce qui, en définitive, a des répercussions négatives sur la textualité (c'est-à-dire sur la continuité du contenu $)^{21}$ (Dalmas, 2005, p. 102).

Dans la production observée, la progression informationnelle est mise en place à travers l'attaque de l'énoncé par l'élément déjà donné ; seul le statut informationnel explique le positionnement et celui-ci se substitue à des explications grammaticales. L'ordre OVS est favorisé ici par la répétition du même schéma récurrent et la reprise de l'élément donné dans l'énoncé précédent est plus économique en termes de ressources que le repérage conscient du placement verbal. Cette réalisation témoigne de la possibilité qu'ont des locuteurs débutants de produire des énoncés spécifiques à la L2 - énoncés dont le traitement syntaxique relève d'un stade avancé mais qui peuvent néanmoins être accessibles par le biais de schémas éprouvés dans l'usage.

\subsection{Une compétence textuelle à développer dès les débuts de l'apprentissage}

Les énoncés des francophones publiés ur le site Kaleidoskop et que nous avons reproduits plus haut trahissent un enseignement qui privilégie la construction correcte de la phrase, c'est-à-dire la morphologie verbale et le positionnement verbal second sans chercher à interroger la mise en texte des phrases produites. Py (2002) déplore que le contexte scolaire

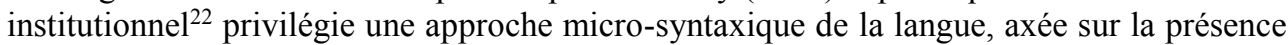
des marques morphosyntaxiques voulues par la norme et accorde peu d'attention à la dimension macro-syntaxique du déploiement informationnel. Dans la tâche tagesablauf, les textes que les apprenants s'avèrent capables de produire à partir d'un input langagier volontairement pauvre témoignent bien d'une planification particulière en amont de la verbalisation - planification qui conduit les apprenants à choisir une organisation informationnelle spécifique en fonction des formes langagières qui sont à leur disposition. La découverte, le repérage et l'usage de ces formes, des schémas dans lesquels elles apparaissent ainsi que du fonctionnement de l'ouverture dans le marquage du décalage constituent dès lors un préalable.

En tant qu'enseignante, nous avons volontairement encouragé les phénomènes de reprise dans les tâches interactives. Elles ont favorisé des linéarisations conformes à la progression informationnelle, mais ont également permis de créer une continuité propositionnelle explicite entre des énoncés successifs qui a pu être exploitée ultérieurement, notamment dans la mise en place de la trame chronologique d'un récit. En effet, au lieu de répéter les adverbiaux temporels donnés dans un énoncé précédent en amorce de l'énoncé suivant, il est possible d'avoir recours à l'expression anaphorique da [là

\footnotetext{
${ }^{21}$ La citation est traduite par nos soins.

${ }^{22}$ Pour des raisons qu'il attribue à une orientation normative de l'école, à la culture grammaticale française et au manque de ressources à disposition des enseignants pour développer une approche macro-syntaxique du discours (Py, 2002, p. 53).
} 
/ à ce moment-là]. De son origine spatiale, ce corrélat a gardé une valeur situative générale qui en fait un élément polyfonctionnel, dont le sens s'interprète différemment en fonction du contexte. Il peut en effet renvoyer à une portion temporelle, spatiale ou encore à un domaine, un avis, des conditions évoquées dans le prétexte. Dans les tâches proposées en première année, cet élément est susceptible d'être introduit à la place d'un adverbial temporel et permet ainsi non seulement de varier les formulations mais aussi de remplir le Vorfeld avec une association Verbe-Sujet automatisée :

(27) (EMA_TAM_kino)

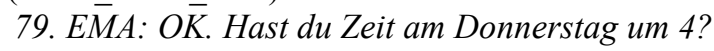

[OK. Tu as du temps jeudi à 4 heures ?]

80. TAM: Unmöglich, [da] habe ich Fußball.

[Impossible, [à ce moment-là] j'ai foot.]

Dans des tâches ultérieures, il est alors utile de rappeler la fonction de $d a$, pour expliquer par exemple le fonctionnement du décalage temporel et des intervalles successifs dans la tâche tagesablauf. Dans l'exemple (12), rappelé ci-dessous, IFI utilise cet élément par deux fois avec des sémantismes qui peuvent s'avérer différents, ce que nous interprétons en faveur d'une sensibilité à la fonction de raccord assumée par les constituants du Vorfeld.

(12) (IFI_tagesablauf)

58. (...) Eine Stunde danach macht sie Hausaufgaben und spielt sie Klavier bis um fünf, da trefft [trifft] sie Freunde aber um sechs soll sie zu Hause sein. Da isst sie nämlich zu Abend, (...)

[(...) Une heure après (ça) elle fait ses devoirs et elle joue du piano jusqu'à 5 heures. Là elle retrouve des amis mais à 6 heures elle doit être à la maison. C'est à cette heure en effet / c'est à la maison en effet qu'elle mange le repas du soir (...)]

Ce qui est plus intéressant encore, c'est qu'une utilisation précoce de cet élément prépare à l'usage de $d a n n^{23}$ [ensuite] et des composés prépositionnels formés à partir de $d a$ tels que $d a+n a c h$ ou $d a+v o r$ [après ça, avant ça] et quand il s'agira d'abord la description spatiale, dar+auf, da+neben, [là-dessus, à côté de ça], etc... Toutes ces expressions fonctionnent à la fois vers l'amont : la composante $d a$ assurant le raccord avec le prétexte et la continuité de sens entre les énoncés, et vers l'aval: la composante prépositionnelle déterminant un repère qui oriente les informations à venir.

A travers l'utilisation de l'ouverture au service la progression informationnelle peuvent se mettre en place des habitudes organisationnelles qui justifient la sélection du constituant préverbal et donnent du sens au positionnement verbal second. Le rôle que jouent les reprises et l'usage des expressions anaphoriques dans l'établissement de la continuité interphrastique est primordial en allemand. Cette fonction spécifique dévolue au Vorfeld recouvre une réalité complexe du fonctionnement de la langue, mais sa mise en œuvre dans des situations d'usage telle que celle décrite ici permet aux apprenants de s'approprier des préférences propres à la L2 qui rendent les énoncés produits « cognitivement » plus proches de ceux produits par des locuteurs natifs.

\section{Conclusion}

Les pratiques langagières au sein de la classe de langue ne sauraient se limiter à formuler des phrases sur la base de prescriptions apprises en amont. Il ne s'agit pas d'actualiser un

${ }^{23}$ Nous considérons avec Blühdorn (2008, p. 20) que le connecteur adverbial dann [ensuite] contient également une dimension anaphorique. 
système dont on aurait acquis les règles de fonctionnement au préalable et les productions d'apprenants, quel que soit leur niveau, témoignent bien souvent de la difficulté à convertir des connaissances déclaratives en connaissances procédurales.

Cortès (2012) souligne le regain d'intérêt pour la question de l'ouverture en allemand suscité par l'ouverture des recherches linguistiques à de nouveaux champs d'investigation. Elle fait notamment référence à des notions relevant de la structure informationnelle des énoncés et de la construction des textes. Ces connaissances théoriques qui décrivent des phénomènes langagiers dans leur fonctionnement ou qui soulignent le rôle de l'usage dans l'appropriation durable de constructions langagières offrent de nouvelles pistes pour la didactique des langues. Ces pistes ont été mises en œuvre dans notre intervention en tant qu'enseignante sur le terrain, et nous avons cherché à en analyser la portée sur les productions des apprenants. Les observations que nous avons menées nous conduisent à considérer la classe de langue comme un lieu d'exploration et d'expérimentation des usages de la langue dans des conditions plus ou moins guidées et contrôlées. Dans ce contexte, l'usage, c'est-à-dire la pratique répétée, contrôlée et explicitée de constructions prototypiques pertinentes dans des situations de communication données, favorise non seulement la mise en place de préférences et d'habitudes plus proches des usages effectifs de la langue cible, mais peut conduire également à l'émergence et à l'appropriation de constructions syntaxiques spécifiques de l'allemand.

Nous remercions le Pr. Dr. Wolfgang Hielber de nous avoir autorisée à reproduire certains extraits issus de contributions postées sur le site Kaleidoskop - Unser Alltag in Deutschland - Und wie ist euer Alltag in ...?

\section{Références}

Ballestracci, S. (2010). Der Erwerb von Verbzweitsätzen mit Subjekt im Mittelfeld bei italophonen DaF-Studierenden. Erwerbsphasen, Lernschwierigkeiten und didaktische Implikationen. Linguistik online, 41(1/10), 25-39.

Blühdorn, H. (2008). Syntax und Semantik der Konnektoren. Ein Überblick. Mannheim: Institut für deutsche Sprache, Manuskript. Consulté à l'adresse http://www1.idsmannheim.de/fileadmin/gra/texte/blu_ueberblick.pdf

Bohnacker, U., \& Rosén, C. (2008). The clause-initial position in L2 German declaratives: Transfer of information structure. In Studies in Second Language Acquisition (Vol. 30). 511-538.

Boss, B., \& Jansen, L. (2003). Do Our Students Learn What We Teach Them? Babel, 38(2), 26-30.

Cedden, G., \& Aydın, Ö. (2007). „Meistens ich weiß nicht, wo ich muss das Verb gebrauchen “. Die Problematik des V2 Phänomens bei türkischen Lernenden der deutschen Sprache. Zeitschrift für Interkulturellen Fremdsprachenunterricht, 12(3). 1-15.

Charolles, M. (2003). De la topicalité des adverbiaux détachés en tête de phrase. Travaux de linguistique, 47(2), 11-49.

Cortès, C. (Éd.). (2012). Satzeröffnung: Formen, Funktionen, Strategien. Tübingen: Stauffenburg Verlag.

Dalmas, M. (2005). Dringend nötig: der «Blick über den Satzrand». Zu den Schwierigkeiten von französischen Studierenden bei der Texterzeugung. In K. Adamzik \& W.-D. Krause (Éd.), Text-Arbeiten. Textsorten im fremd-und muttersprachlichen Unterricht an Schule und Hochschule. Tübingen: Gunter Narr Verlag. 97-109. 
Diehl, E., Christen, H., Leuenberger, S., Pelvat, I., \& Studer, T. (2000). Grammatikunterricht: Alles für der Katz?: Untersuchungen zum Zweitsprachenerwerb Deutsch. Tübingen: Niemeyer.

Ellis, R. (1989). Are classroom and naturalistic acquisition the same? Studies in Second Language Acquisition, 11(03), 305-328.

Ellis, R. (2003). Task-based Language Learning and Teaching. Oxford: Oxford University Press.

Fabricius-Hansen, C. (2010). Syntax. In H.-J. Krumm, C. Fandrych, B. Hufeisen, \& C. Riemer (Éd.), Deutsch als Fremd- und Zweitsprache. Ein internationales Handbuch (Vol. 1). Berlin, New York: De Gruyter Mouton. 216-226.

Felce, C. (2015, novembre 30). L'ouverture de l'énoncé en allemand L2: de la compréhension d'un phénomène à son appropriation et à son enseignement. Perspectives en didactique des langues (Thèse de doctorat en sciences du langage, sous la direction de D. Macaire et de D. Véronique). Paris 3 - Sorbonne Nouvelle, Paris.

Lahousse, K. (2003). La complexité de la notion de topique et l'inversion du sujet nominal. Travaux de linguistique, $n^{\circ} 47(2), 111-136$.

Legallois, D., \& François, J. (2011). La Linguistique fondée sur l'usage : parcours critique. Travaux de linguistique, 62(1), 7-7.

Levelt, W. J. M. (1989). Speaking: From Intention to Articulation. Cambridge: MIT Press.

Marschall, G., R. (2012). Einstieg, Fortführung, Neuorientierung - Satzanfänge als Indizien für Diskurskonstruktion. In C. Cortès (Éd.), Satzeröffnung: Formen, Funktionen, Strategien. Tübingen: Stauffenburg Verlag. 157-180.

Meisel, J. M., Clahsen, H., \& Pienemann, M. (1981). On determining developmental stages in natural second language acquisition. Studies in Second Language Acquisition, 3(02), 109 -135 .

Muranoi, H. (2007). Output Practice in the L2 classroom. In R. DeKeyser M. (Éd.), Practice in a Second Language: Perspectives from Applied Linguistics and Cognitive Psychology. Cambridge University Press. 51-84.

Pienemann, M. (1984). Psychological Constraints on the Teachability of Languages. Studies in Second Language Acquisition, 6(02), 186-214.

Pienemann, M. (1989). Is language teachable? Psycholinguistic experiments and hypotheses. Applied Linguistics, 10(1), 52-79.

Pienemann, M. (1999). Language processing and second language development: processability theory (Vol. 1-1). Amsterdam: J. Benjamins.

Pienemann, M., Keßler, J.-U., \& Itani-Adams, Y. (2011). Comparing levels of processability across languages. International Journal of Bilingualism, 15(2), 128-146.

Poitou, J. (2010). Fiches de grammaire allemande. Consulté 7 mai 2013, à l'adresse http://j.poitou.free.fr/pro/html/div/fiches.html

Py, B. (2002). Acquisition d'une langue seconde, organisation macrosyntaxique et émergence d'une microsyntaxe. Marges linguistiques, 1(4), 48-55.

Schmidt, T., \& Wörner, K. (2009). EXMARaLDA - Creating, analysing and sharing spoken language corpora for pragmatic research. Pragmatics, 19(4), 565-582. 
Skehan, P. (2007). Language Instruction Through Tasks. In J. Cummins \& C. Davison (Éd.), International Handbook of English Language Teaching (Vol. 15)). Springer US. 289 -301 .

Speyer, A. (2008). German Vorfeld-filling as constraint interaction. In A. Benz \& Peter Kühnlein (Éd.), Constraints in Discourse . John Benjamins Publishing Company. 267-290.

Véronique, D. (Éd.). (2009). L'acquisition de la grammaire du français, langue étrangère (Didier). Paris.

Vinckel-Roisin, H. (2012). Von Anfang bis Ende: Referenzketten, Textgliederung und Syntax im Online-Journalismus. In C. Cortès (Éd.), Satzeröffnung: Formen, Funktionen, Strategien. Stauffenburg Verlag. 221-233.

von Stutterheim, C. (1997). Einige Prinzipien des Textaufbaus: Empirische Untersuchungen zur Produktion mündlicher Texte. Tübingen: Niemeyer.

von Stutterheim, C., \& Carroll, M. (2005). Subjektwahl und Topikkontinuität im Deutschen und im Englischen. Zeitschrift für Literaturwissenschaft und Linguistik, 139, 7-27.

\section{Manuels scolaires cités}

Esterl, U., Körner, E., \& Einhorn, A. (2008). Team Deutsch 1. Maison des langues.

Goldmann, K., Jacqueroud, U., \& Robert, J. (2013). Kreativ. Allemand. palier 1. Année 1. Hachette Education.

Haldenwang, N., Lozachmeur, F., Pavan, C., \& Suissa, P. (2009). Gute Fahrt. Allemand lère année. Nouveaux programmes. Nathan.

Faure-Paschal, N., Hoock-Douilly, M., Rakotondravao, C., \& Wegener, A. (2013). Spontan Ineu, palier 1, Ire année. Didier. 